\title{
Split-ring polariton condensates as macroscopic two-level quantum systems
}

\author{
Yan Xue $\odot,,^{1,2,3, *}$ Igor Chestnov $\odot,{ }^{1,3,4, \uparrow}$ Evgeny Sedov, ${ }^{1,3,4}$ Evgeniy Kiktenko, ${ }^{5,6}$ Aleksey K. Fedorov, ${ }^{5,6}$ \\ Stefan Schumacher, ${ }^{7}$ Xuekai Ma, ${ }^{7, *}$ and Alexey Kavokin $\oplus^{1,3,5,8}$ \\ ${ }^{1}$ School of Science, Westlake University, 18 Shilongshan Road, Hangzhou 310024, Zhejiang Province, China \\ ${ }^{2}$ College of Physics, Jilin University, Changchun 130012, China \\ ${ }^{3}$ Institute of Natural Sciences, Westlake Institute for Advanced Study, 18 Shilongshan Road, Hangzhou 310024, Zhejiang Province, China \\ ${ }^{4}$ Vladimir State University, Gorkii St. 87, 600000, Vladimir, Russia \\ ${ }^{5}$ Russian Quantum Center, Skolkovo, Moscow 143025, Russia \\ ${ }^{6}$ Moscow Institute of Physics and Technology, Dolgoprudny 141700, Russia \\ ${ }^{7}$ Department of Physics and Center for Optoelectronics and Photonics Paderborn (CeOPP), Universität Paderborn, \\ Warburger Strasse 100, 33098 Paderborn, Germany
}

(Received 26 March 2020; revised 14 July 2020; accepted 5 January 2021; published 29 January 2021)

\begin{abstract}
Superposition states of circular currents of exciton-polaritons mimic the superconducting flux qubits. The phase of a polariton fluid must change by an integer number of $2 \pi$ when going around the ring. If one introduces a $\pi$-phase delay line in the ring, the fluid is obliged to propagate a clockwise or anticlockwise circular current to reduce the total phase gained over one round trip to zero or to build it up to $2 \pi$. We show that such a $\pi$-delay line can be provided by a dark soliton pinned to a potential well created by a C-shape nonresonant pump spot. The resulting split-ring polariton condensates exhibit pronounced coherent oscillations passing periodically through clockwise and anticlockwise current states. These oscillations may persist far beyond the coherence time of polariton condensates. The qubits based on split-ring polariton condensates are expected to possess very high figures of merit that makes them a valuable alternative to superconducting qubits. The use of the dipole-polarized polaritons allows one to control coherently the state of the qubit with the external electric field. This is shown to be one of the tools for realization of single-qubit logic operations. We propose the design of an iSWAP gate based on a pair of coupled polariton qubits. To demonstrate the capacity of the polariton platform for quantum computations, we propose a protocol for the realization of Deutsch's algorithm with polariton qubit networks.
\end{abstract}

DOI: 10.1103/PhysRevResearch.3.013099

\section{INTRODUCTION}

While tremendous progress in the development of quantum technologies is apparent, it is still unclear which material platform is the most suitable for the realization of future quantum computers and simulators [1]. Among the leaders of the quest are superconducting circuits with Josephson junctions [2-7], cold atoms in optical traps [8-10], ions [11-13], and purely photonic systems [14], which already provide computing facilities on the border of the capabilities of classical computing devices. The semiconductor platform lags slightly behind so far, while remarkable progress has been recently achieved [15] with spin-based quantum computing in semiconductor nanostructures [16,17] as well as in the creation of singlephoton sources based on quantum dots [18]. Recently, a series

\footnotetext{
*xy4610@jlu.edu.cn

†igor_chestnov@westlake.edu.cn

¥xuekai.ma@gmail.com

§a.kavokin@westlake.edu.cn
}

Published by the American Physical Society under the terms of the Creative Commons Attribution 4.0 International license. Further distribution of this work must maintain attribution to the author(s) and the published article's title, journal citation, and DOI. of papers demonstrated a high potentiality of semiconductor microcavities in the strong light-matter coupling regime for hosting ensembles of phase-locked bosonic condensates of half-light-half-matter quasiparticles: exciton polaritons (hereafter referred to as polaritons for brevity) $[19,20]$. It has been argued that the phase-locking process in an array of polariton condensates may be used for the minimization of a classical many-body $X Y$ Hamiltonian [20]. Polariton condensates may be formed at elevated temperatures, optically controlled and mutually phase locked on a picosecond timescale. These features constitute their main potential advantages over other material platforms for realization of quantum simulators. On the other hand, a polariton qubit has never been convincingly demonstrated till now, and it has been argued that the dissipative nature of exciton polaritons characterized by ultrashort radiative lifetimes would prevent their use for implementations of quantum algorithms [21]. Quite recently, an interesting proposal [22] to build the qubit on quantized fluctuations of the resonantly driven polariton condensate in the cylinder microcavities was made.

Here we propose a quite different approach. In particular, we argue that a strong fundamental similarity of superfluid polariton flows [23] and superconducting electric currents may be exploited to build a polariton analog of the superconducting flux qubit. Superconducting flux qubits are based 


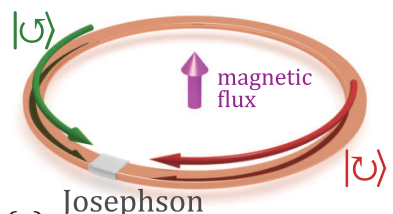

(a) junction

(c)
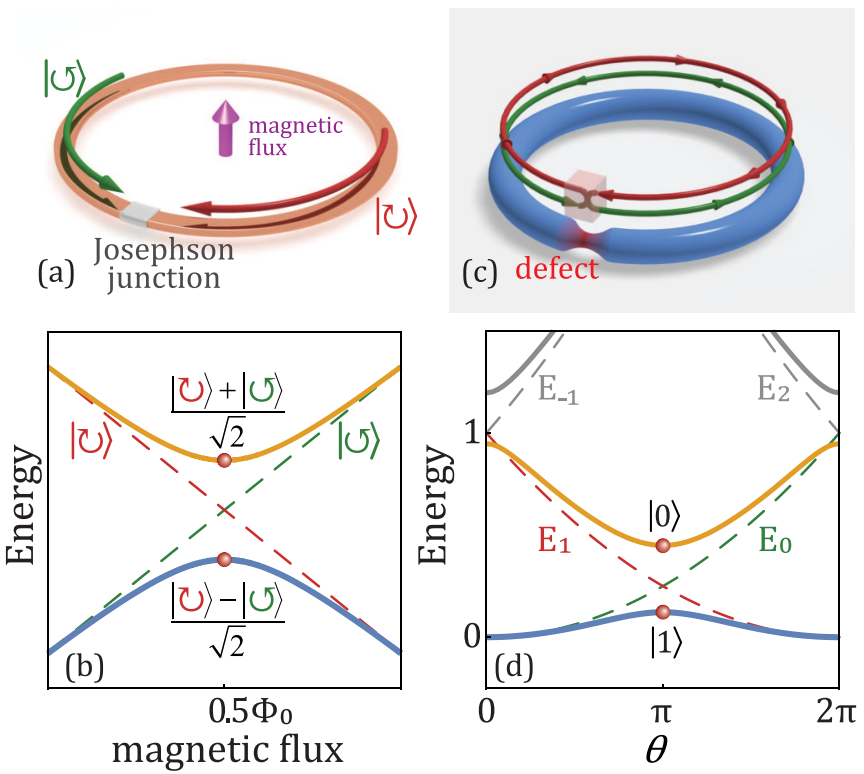

FIG. 1. Comparison of a superconducting flux qubit and a splitring polariton condensate qubit. (a) Sketch of a flux qubit consisted of a superconductor circuit interrupted by a Josephson junction. The persistent currents generated inside the loop tend either to compensate an external magnetic flux or build it up to the value corresponding to the full magnetic flux quantum $\Phi_{0}(|\circlearrowright\rangle$ and $|\circlearrowleft\rangle$ current states, respectively). (b) Energy levels of a flux qubit. The qubit basis is formed by symmetric and antisymmetric superpositions of the persistent current states. (c) Loop of a polariton superfluid with an embedded defect. (d) Energy diagram of the states of the superfluid circle with different topological charges $l$ in the presence of the effective magnetic field. The energy of the state, $E_{l}=$ $E_{1}(\theta=0)[l-\theta / 2 \pi]^{2}$, is measured in units of $E_{1}(\theta=0)$.

on superpositions of clockwise and anticlockwise currents formed by millions of Cooper pairs [24-26]; see Figs. 1(a) and 1(b). In order to excite the system in a superposition state, the half-quantum flux of magnetic field is passed through the superconducting circuit containing one or several Josephson junctions. The system is forced to generate a circular current to either reduce the magnetic flux to zero or to build it up to a full-quantum flux.

While electrically neutral polaritons are much less sensitive to the external magnetic field [27] than Cooper pairs, the circular currents of superfluid polaritons [28] can be efficiently controlled by introducing a potential defect (a phase delay line) in a polariton ring. The defect couples counterpropagating polariton currents. This results in a formation of a two-level quantum system based on a split-ring polariton condensate. One of the efficient methods for the realization of such a defect implies pinning a dark soliton [29,30], which is characterized by a $\pi$-jump of phase of a superfluid, to the slot in the polariton ring. The $\pi$-phase delay line embedded in a circle forces the superfluid to flow clockwise or anticlockwise in order to either build up the phase variation along the loop to $2 \pi$ or to reduce it to zero. We run numerical experiments showing the spontaneous excitation of robust oscillations of the resulting polariton qubit state on the Bloch sphere; see Sec. II C. These oscillations are caused by the continuous phase change between the qubit basis states. They are indicative of the formation of the macroscopic superposition of the qubit basis states corresponding to the symmetric and antisymmetric combinations of polariton circular currents.

Interestingly, the predicted dephasing time of the oscillations in such a system nonresonantly pumped by a continuous-wave $(\mathrm{CW})$ laser source appears to be orders of magnitude longer than the characteristic oscillation period (about $125 \mathrm{ps)}$ ). This may result in very high figures of merit of qubits based on split-ring polariton condensates. This is because the phase gradient that governs the current states of polariton condensates is insensitive to the overall timedependent phase characterizing the condensate as a whole object. Since the condensate state is continuously sustained by the external pumping, the system reaches the regime of dynamic equilibrium, which is manifested by the dynamical balance between gain and dissipation. Therefore, the circular polariton currents are expected to survive as long as the pumping is switched on [31].

As a first step towards realization of polariton quantum networks, we propose a design of the gates performing the Pauli rotation operations which allows for the manipulation of the state of an individual qubit. The proposed solution implies the use of the gauge field capable of lifting the degeneracy between the opposite persistent currents by additionally breaking the time-reversal symmetry. For this purpose, we propose to employ a synthetic gauge field arising from the coupling between the motion of dipole-polarized excitons and the magnetic field [32]. This approach allows for the realization of a coherent control between the qubit basis states.

For realization of a multiqubit quantum processing, we design the $i$ SWAP gate based on two coupled split-ring polariton condensates. In order to demonstrate a high potentiality of semiconductor microcavities for designing of the quantum information devices, we test the system for implementability of the practical quantum protocols. As a benchmark test, we simulate Deutsch's algorithm, whose realization is typically recognized as a necessary step for demonstration of the feasibility of the quantum information processing on a given material platform. This was the case of the early experiments with nuclear magnetic resonance systems [33,34] as well as with the advanced platforms, such as trapped ions [35], superconducting circuits [36], and photonic systems [37,38]. Implementation of Deutsch's algorithm requires the realization of both single- and two-qubit gates. The demonstration of this algorithm then turns to a proof-of-concept test of the ensamble of required tools for further scaling quantum information processing systems since an arbitrary quantum algorithm can be decomposed in a sequence of single- and two-qubit gates.

\section{RESULTS}

\section{A. The origin of the two-level quantum system}

Let us consider a close circuit filled with a coherent quantum fluid. The phase of the many-body wave function $\psi(t, s)$ of the fluid $\varphi$ must obey the equality $\oint_{D} \partial_{s} \varphi d s=2 \pi \ell$, which is the quantization condition for the topological invariant $\ell \in$ $\mathbb{Z}$ also known as the winding number $[39,40]$. Here $s$ is the 

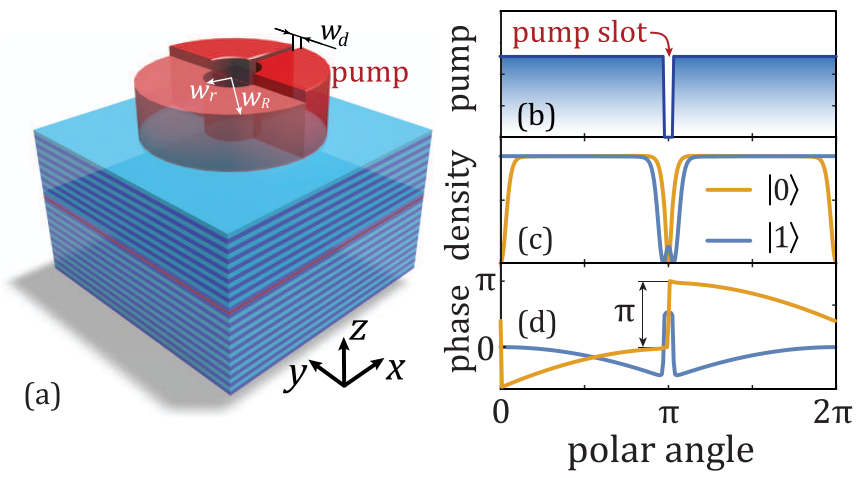

FIG. 2. The model system. (a) The semiconductor microcavity excited by a nonresonant pump beam having the $\mathrm{C}$-shape profile. Here $w_{r}=6 \mu \mathrm{m}$ and $w_{R}=20 \mu \mathrm{m}$. The width of the slot is $w_{d}=$ $2 \mu \mathrm{m}$. (b)-(d) Properties of the basis states of the qubit formed in a polariton superfluid ring, as predicted by the 1D model. (b) The pump distribution contains a slot which is able to pin a dark soliton manifesting itself in the density dip and the $\pi$ phase jump (d). (c) The densities and (d) the phases of the basis states $|0\rangle$ and $|1\rangle$. (c), (d) The solutions of the $1 \mathrm{D}$ problem obtained with a pump distribution shown in (b). The pump amplitude is $3.5 \times P_{\mathrm{th}}$.

coordinate along the circuit of a total length $D$. If the fluid is subjected to the gauge field, characterized by the vector potential $\mathbf{A}$, the quantization condition becomes $\oint_{D} \partial_{s} \varphi d s-$ $\theta=2 \pi \ell$. The phase delay $\theta=\Lambda \Phi / \hbar$ induced by the vector field is determined by its flux $\Phi$ and the constant $\Lambda$ which defines the pulse rescaling rule, $\hat{\mathbf{p}} \rightarrow \hat{\mathbf{p}}-\Lambda \mathbf{A}$. The effective flux governs the energy spectrum of a two-level system based on counterpropagating currents with opposite winding numbers, as Fig. 1(d) shows. At the particular value $\theta=\pi$, the states with $\ell=0$ and $\ell=1$ are degenerate in energy similar to the case of the superconducting flux qubit. These states, however, are different in the phase increment $\oint_{D} \partial_{s} \varphi d s$, which equals $\pi$ for the state with zero topological charge $\ell=0$ and $-\pi$ for the state with $\ell=1$.

The energy gap between the $\ell=0$ and $\ell=1$ states appears in the presence of a defect embedded in a circuit; see Figs. 1(c) and 1(d). The defect causes back-scattering of the currents and mixes them. The eigenstates of this system mimic the linear superposition states realized in a superconducting flux qubit.

For electrically neutral particles the phase delay $\theta$ can be engineered either by the circular motion of the defect [41] or by exploiting the spin-orbit coupling in the presence of external magnetic fields [27]. However, the specific case of $\theta=\pi$ can be realized in a much simpler way. All one has to do is to embed at some point a dark soliton state characterized by a $\pi$-jump of the phase. This can be done, e.g., by pinning it with a potential defect required for localization and stabilization of the soliton state $[42,43]$. In the presence of a dark soliton, the current states with the phase changing by $\pi$ and $-\pi$ over the remaining part of the circuit form two superposition states $|0\rangle$ and $|1\rangle$, which constitute a two-level quantum system or qubit.

\section{B. The model}

We consider the system shown schematically in Fig. 2(a). A semiconductor microcavity formed by a couple of Bragg mirrors contains an ensemble of embedded quantum wells. The strong coupling of cavity photons and quantum-well excitons results in the appearance of new eigenmodes of the structure, the exciton polaritons [44]. We assume that the polaritons are created by the nonresonant $\mathrm{CW}$ optical pump of a C-shape, which is schematically illustrated in Fig. 2(a). Obeying the bosonic statistics, polaritons form a Bose-Einstein condensate which remains localized under the pump spot due to the finite polariton lifetime.

The dynamics of a $2 \mathrm{D}$ split-ring polariton condensate in a semiconductor microcavity obeys the driven-dissipative Gross-Pitaevskii equation coupled with the rate equation for the density of the exciton reservoir:

$$
\begin{aligned}
d \psi(\mathbf{r})= & \left\{\frac{i \hbar}{2 m^{*}} \nabla^{2}-\frac{i}{\hbar}\left[g_{c}|\psi(\mathbf{r})|^{2}+g_{r} n_{R}(\mathbf{r})+V(\mathbf{r})\right]\right. \\
& \left.+\frac{1}{2}\left[R n_{R}(\mathbf{r})-\gamma_{c}\right]\right\} \psi(\mathbf{r}) d t+d W, \\
\frac{\partial n_{R}(\mathbf{r})}{\partial t}= & P(\mathbf{r})-\left[\gamma_{R}+R|\psi(\mathbf{r})|^{2}\right] n_{R}(\mathbf{r}),
\end{aligned}
$$

where $\psi(\mathbf{r})$ is a polariton condensate order parameter, $n_{R}(\mathbf{r})$ is the exciton reservoir density, $m^{*}=10^{-4} m_{e}$ is the effective mass of polaritons on the lower branch $\left(m_{e}\right.$ is the free electron mass), and $\gamma_{c}=1 / 6 \mathrm{ps}^{-1}$ and $\gamma_{R}=2 \gamma_{c}$ are the polariton and the reservoir decay rates, respectively. $R=0.01 \mathrm{ps}^{-1} \mu \mathrm{m}^{2}$ is the rate of the stimulated scattering from the exciton reservoir to the polariton condensate. $g_{c}=6 \mu \mathrm{eV} \mu \mathrm{m}^{2}$ and $g_{r}=2 g_{c}$ describe the interaction of polaritons between themselves and with the reservoir excitons, respectively. $V(\mathbf{r})$ is the external potential. The chosen values of the parameters of the model are relevant to the experimental data [45].

While the classical fluctuations are taken into account in the initial polariton field, the effect of quantum fluctuations is included by adding a complex stochastic term within the truncated Wigner approximation [46]

$$
\begin{aligned}
\left\langle d W(\mathbf{r}, t) d W\left(\mathbf{r}^{\prime}, t\right)\right\rangle & =0, \quad\left\langle d W(\mathbf{r}, t) d W\left(\mathbf{r}, t^{\prime}\right)\right\rangle=0, \\
\left\langle d W(\mathbf{r}, t) d W^{*}\left(\mathbf{r}^{\prime}, t^{\prime}\right)\right\rangle & =\frac{d t}{2 d x d y}\left(R n_{R}+\gamma_{c}\right) \delta_{\mathbf{r}, \mathbf{r}^{\prime}} \delta_{t, t^{\prime}} .
\end{aligned}
$$

The CW nonresonant pump $P(\mathbf{r})$ of a $\mathrm{C}$-shape, as shown in Fig. 2(a), is characterized by the spatial distribution of the intensity given by

$$
P(\mathbf{r})= \begin{cases}P_{0}\left[1-e^{-\left(\frac{\mathbf{r}}{w_{r}}\right)^{n}}\right]\left[1-e^{-\left(\frac{2 y}{w_{d}}\right)^{n}}\right] e^{-\left(\frac{\mathbf{r}}{w_{R}}\right)^{n}}, & x>0, \\ \left(P_{0}-P_{1}\right)\left[1-e^{-\left(\frac{\mathbf{r}}{w_{r}}\right)^{n}}\right] e^{-\left(\frac{\mathbf{r}}{w_{R}}\right)^{n}}, & x \leqslant 0,\end{cases}
$$

where the pump power variation factor $P_{1}$ quantifies the depth of the step in the pump beam intensity as shown in Fig. 2(a). By additionally breaking the rotation symmetry, this step allows for manipulation of the quantum properties of the two-level system, in particular, for switching between the superposition and the pole states, as will be demonstrated hereafter. The steepness parameter is taken as $n=20$. The required profile of the pump beam can be realized using spatial light modulator $[19,28]$ or by pattering the microcavity surface with an opaque mask intended to prevent excitation of polaritons beneath it. 
The key ingredient of the pump is a radial slot of the width $w_{d}$ where no polaritons are excited. Its function is to mix the opposite polariton currents giving rise to the energy splitting between the basis states. As well, it favors formation of the soliton-like topological defect in the polariton condensate accompanied by the $\pi$ jump of the phase in the azimuthal direction. In particular, due to the local absence of the gain, the polariton density is typically depleted at the position of the slot. It naturally triggers formation of the dark soliton provided that the polariton-polariton interactions are strong enough to sustain it [42]. Besides, the reservoir density also appears to be depleted under the slot. Since excitons repel each other, the slot is responsible for the formation of the potential well for the condensate, which serves for pinning and stabilization of the soliton state.

In the presence of the slot, in addition to the persistent current states with nonzero average momenta [47], the couple of energy nondegenerate states with zero average currents appears. These are symmetric and antisymmetric states similar to those presented in Fig. 1(d). The typical angular dependencies of the magnitudes and the phases of the corresponding wave functions are shown in Figs. 2(c) and 2(d). The properties of these states can be conveniently analyzed in the limit where the pump profile is a thin ring of a large radius; see Appendix B. The basis states behave differently in the vicinity of the defect, which causes splitting in their energies. Therefore, the value of the energy gap between these states can be controlled by both shape and intensity of the pump as is discussed in Appendix B.

\section{The dynamics of a split-ring condensate}

In what follows, we shall focus on the oscillatory regime of a $2 \mathrm{D}$ split-ring condensate. In this regime, the condensate is initially formed in a superposition of $|0\rangle$ and $|1\rangle$ eigenstates $[48,49]$. The system exhibits long-living quantum beats whose frequency is governed by the energy splitting of $|0\rangle$ and $|1\rangle$ states. The split-ring condensate passes periodically through clockwise and anticlockwise current states. Its dynamics can be conveniently mapped to a Bloch sphere. At this stage we neglect by the quantum fluctuations of the condensate order parameter focusing on its coherent dynamics.

Figure 3 shows the oscillatory regime of the split-ring polariton condensate predicted by the numerical modeling of Eqs. (1). We characterize nonstationary circular current states of the condensate by the normalized average angular momentum $m(t)=\hbar^{-1} L_{z}(t) / N(t)$, where $L_{z}(t)=$ $(-i \hbar) \int \psi^{*}(\mathbf{r}, t)\left[x \partial_{y}-y \partial_{x}\right] \psi(\mathbf{r}, t) d \mathbf{r}$ is the actual average angular momentum and $N(t)=\int|\psi(\mathbf{r}, t)|^{2} d \mathbf{r}$ is the number of polaritons in the condensate. In contrast to the winding number $\ell$, the average angular momentum $m(t)$ continuously varies in the course of the evolution of the condensate and can have arbitrary real value. The oscillations of the polariton state in Fig. 3(a) occur between the states with the average angular momenta of $m \simeq 0.4$ and $m \simeq-0.4$. Figures $3(\mathrm{~b})$ and $3(\mathrm{~d})$ show the intensity distribution (left), the phase distribution in the cavity plane (middle), and the angular phase distribution (right) of the polariton states with $m \simeq 0.4$ and $m \simeq-0.4$, respectively. Figure 3(c) illustrates the intermediate state of $m=0$ visited by the system in the course of the oscillations.
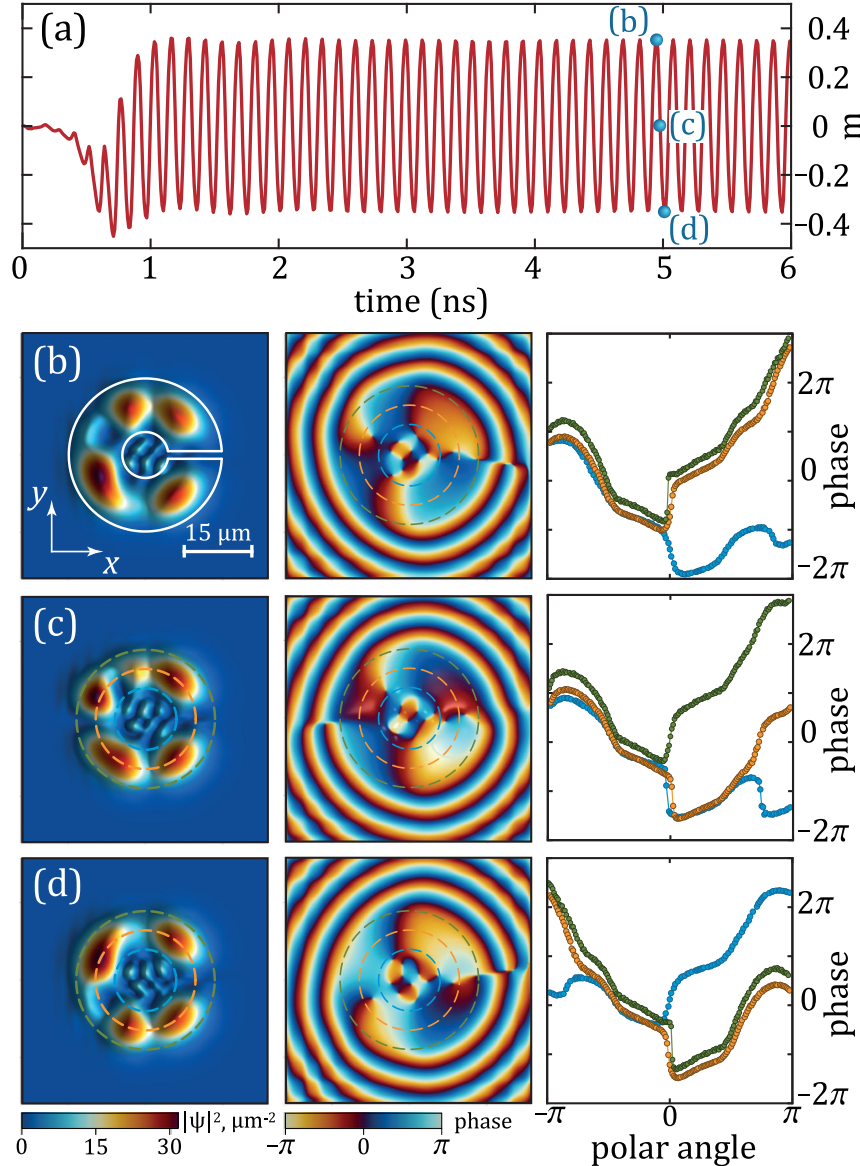

FIG. 3. The temporal evolution of the split-ring polariton condensate nonresonantly pumped with the $\mathrm{C}$-shape $\mathrm{CW}$ laser beam switched on at time zero. (a) The temporal evolution of the mean angular momentum $m$ of the condensate. (b)-(d) The density (left column) and the phase (middle column) profiles are shown in (b)(d) for the states with the maximum, zero, and minimum values of the angular momentum, respectively. The pump shape is indicated with a white contour in panel (b). The phase of the condensate as a function of the polar angle at the fixed radius $r$ is shown in the right column. Here $r=8 \mu \mathrm{m}, 13 \mu \mathrm{m}$, and $18 \mu \mathrm{m}$ for blue, orange, and green curves, respectively. The parameters of the pump beam used for this calculation are $P_{0}=1.8 \times P_{\text {th }}$ and $P_{1}=0.1 \times P_{0}, P_{\text {th }}=\gamma_{c} \gamma_{R} / R$.

The full dynamics of the oscillations illustrated by Fig. 3 is summarized in the Supplemental Movie [50].

The harmonic oscillations of the angular momentum of the condensate can be considered as a fingerprint of a many-body two-level quantum system. The spectral analysis of the oscillatory dynamics of the system reveals two sharp resonances appearing, which are split in energy by about $32 \mu \mathrm{eV}$ (see Appendix D and Fig. 12), which corresponds to the period of the oscillations seen in Fig. 3(a).

Figure 4 shows the trajectory of the quantum state of the split-ring polariton condensate on the surface of the Bloch sphere based on $|0\rangle$ and $|1\rangle$ eigenstates (see details of the mapping in Appendix A). Retaining only the classical fluctuations of the initial polariton field and neglecting the stochastic processes [Figs. 3 and 4(a)], we obtain the stable oscillations that persist during over $10 \mathrm{~ns}$ (truncation time of this numerical 


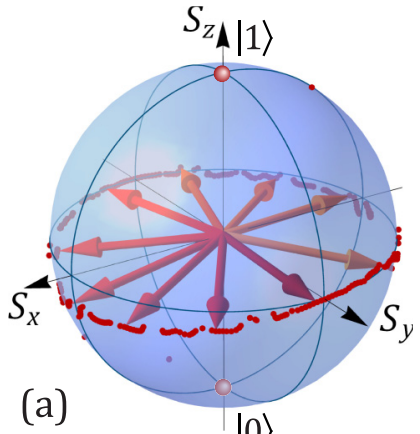

$|0\rangle$

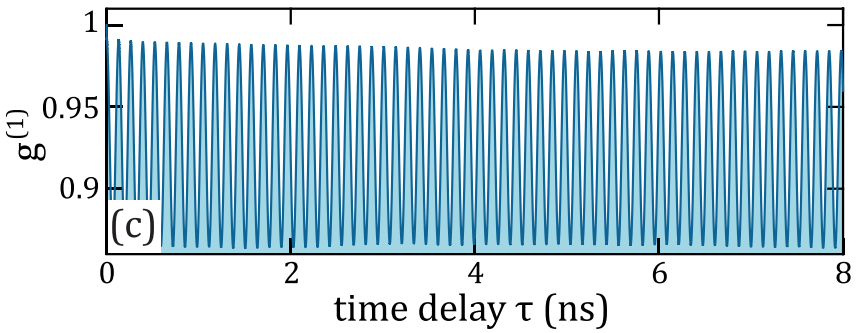

FIG. 4. The evolution of the quantum state of the split-ring polariton condensate on the surface of the Bloch sphere. Four periods of oscillations are shown. (a) Computed neglecting and (b) accounting for the quantum fluctuations introduced by Eq. (2). (c) First-order coherence $g^{(1)}$ as a function of time delay $\tau$ corresponding to the dynamics shown in panel (b). The decay of the envelope is caused by the presence of fluctuations. The parameters are the same as in Fig. 3.

simulation) and correspond to a circular trajectory close to the equator of the Bloch sphere. When including the quantum fluctuations described by Eq. (2), we find that the uncertainty in the mapping procedure to the Bloch sphere becomes larger and the trajectory of our system on the surface of the Bloch sphere looks noisy. Remarkably, the noise does not affect the stability of oscillations that persist over the whole calculation period showing no apparent decay. This is because the oscillating state is continuously sustained by the pump. It can be characterized as a regime of the dynamical equilibrium where the losses are exactly compensated by the time averaged gain. We conclude that the harmonic oscillations in a two-level quantum system formed by a split-ring polariton condensate may persist as long as the pumping is on.

The reason for the surprising stability of oscillations is in the topological protection of circular current states in splitring condensates. The superfluid currents are governed by the spatial distribution of the phase of the condensate. The localization radius of the condensate ( $20 \mu \mathrm{m}$ in our case) is much smaller than the coherence length in the system (over $100 \mu \mathrm{m}$ ) [51], which is why the coherence of superfluid polariton condensate is preserved. The coherence time of the condensate as a whole which characterizes the time dependence of the overall phase of the ring condensate does not impose limitations on the lifetime of polariton currents. Note that persistent circular currents in polariton superfluids have been experimentally observed recently [51-53].

The stable persistent oscillations of the polariton quantum system in the vicinity of the equator of the Bloch sphere are

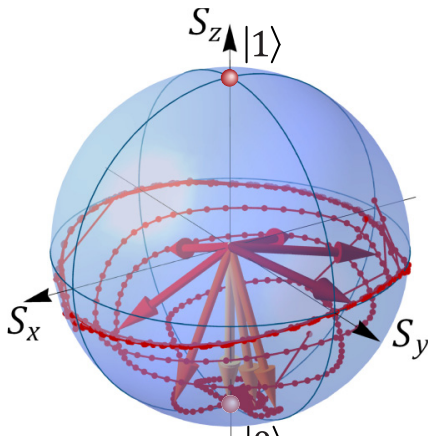

(a)

$|0\rangle$

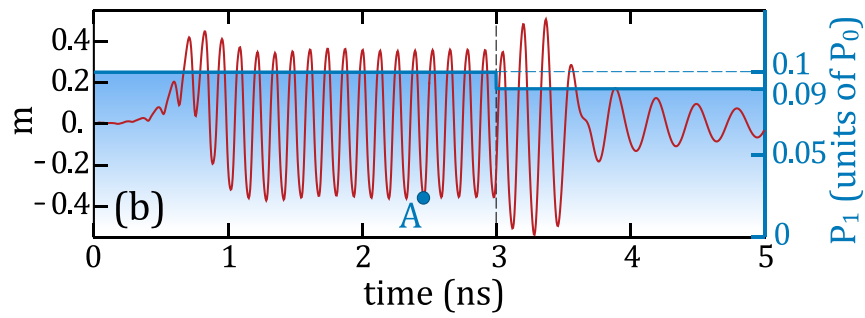

FIG. 5. The oscillations of the quantum state of the split-ring condensate. The decay that is controlled by $P_{1}: P_{1}=0.1 \times P_{0}$ while $t<3$ ns and $P_{1}=0.09 \times P_{0}$ while $t>3$ ns. (a) The trajectory of the quantum system on the Bloch sphere shown from point $\mathrm{A}$ on the time axis onward; (b) the dynamics of the angular momentum of the condensate. The switch of $P_{1}$ occurs at $t=3 \mathrm{~ns}$ (vertical dashed line).

observed if the parameter $P_{1}$ characterizing the step in the pump power distribution [see Eq. (3)] is chosen in the range of $[0.1,0.3] \times P_{0}$. With the decrease of $P_{1}$ down to $0.09 \times P_{0}$, the stability of the oscillations is broken and the system exhibits a fast decay. Figure 5 shows the variation of the dynamics of the system resulting from the variation of the value of $P_{1}$ : while $t$ is shorter than $3 \mathrm{~ns}$, the system exhibits the same stable oscillations as those shown in Figs. 3 and 4. Next, as a result of the decrease of $P_{1}$ from $0.1 \times P_{0}$ to $0.09 \times P_{0}$ at $t=3 \mathrm{~ns}$, the fast decay of the oscillations is observed, so that, eventually, the system relaxes to one of the eigenstates, specifically, to the state $|0\rangle$ shown in Fig. 6(a). We emphasize that the decay time of oscillations is still independent of the coherence time of the condensate in this regime. It is fully governed by $P_{1}$ parameter that controls the magnitude of the step potential. The trajectory of the system on the Bloch sphere that describes the decay of the oscillations has been smoothed with use of the Bezier function [54] for clarity.

One can see in Fig. 6(a) that the wave function of the split-ring condensate is antisymmetric with respect to the horizontal axis $(y=0)$, which passes through the center of the slot. A $\pi$ phase jump appears at $y=0$. With the further decrease of $P_{1}$ down to $0.03 \times P_{0}$, the system relaxes to the basis state $|1\rangle$ shown in Fig. 6(b). It represents the symmetric pattern with a pair of $\pi$ phase jumps close to the horizontal axis $(y=0)$. Note that both basis states are characterized by zero average polariton flow, $m=0$, and represent $2 \mathrm{D}$ counterparts of the basis states shown in Figs. 2(c) and 2(d). Also, they are nearly perfectly orthogonal. Their orthogonality is essential for mapping the system to the Bloch sphere.

It is important to note that, in a general case, the ring condensate is not expected to relax to the lowest energy state 


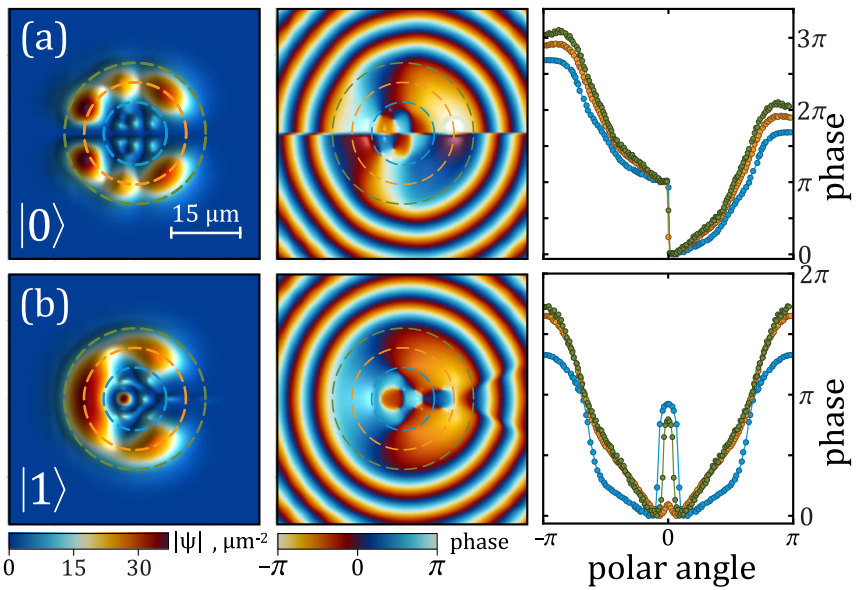

FIG. 6. Two basis states of the split-ring polariton qubit corresponding to the two poles of the Bloch sphere: $|0\rangle$ and $|1\rangle$. (a) $P_{1}=$ $0.09 \times P_{0}$ and (b) $P_{1}=0.03 \times P_{0}$. The density and phase profile in the $2 \mathrm{D}$ case are shown in the left and middle panels, respectively; the angular dependencies of the phase corresponding to different fixed radii are shown with different colors in the right panels $(r=$ $8 \mu \mathrm{m}, 13 \mu \mathrm{m}$, and $18 \mu \mathrm{m}$ are shown with blue, orange, and green, respectively).

corresponding to the upper pole of the Bloch sphere. This is a characteristic feature of polariton lasers: out of all quantum states the system chooses one that maximizes the occupation number of the condensate, but not necessarily one that is characterized by the lowest energy [19,20]. This is why incoherent processes of acoustic-phonon assistant energy relaxation are not expected to affect the dynamics of qubits based on splitring polariton condensates.

\section{C-shape potentials}

Till now we were considering the polariton condensates imprinted in a planar microcavity by means of the optical pumping. Their spatial localization was imposed by the shape of the nonresonant pump used for their excitation. An alternative way to realize split-ring polariton condensates is by using laterally confined $\mathrm{C}$-shape potentials produced by chemical etching of planar cavities [55]. In this case, we expect a stronger confinement of polaritons and more control tools for shaping the condensates. The drawback of this system as compared to fully optically induced split-ring condensates is in its rigidity: each time, to change the geometry of an array of polariton condensates one would need to grow a new sample. We also consider the combined method of lateral confinement by using the etched micropillars where ring condensates are formed due to the repulsion of polaritons from the exciton reservoir formed in the center of the pillar by a nonresonant optical pumping. Persistent superfluid currents of exciton polaritons were recently observed in such structures [52]. Shifting the pump spot from the center of the pillar yields the formation of the split-ring condensate $[53,56]$. The results of numerical simulations of the harmonic oscillations in polariton condensates confined to $\mathrm{C}$-shape potentials are shown in Appendix C.
We note that a qualitatively similar phenomenology was recently revealed in optical vortices and circular Bose-Einstein condensates [57-59]. We are confident that the polariton platform possesses a number of important advantages for the realization of quantum networks as compared to the systems studied in these works. One of the most important advantages comes from the use of semiconductor structures for which growth and fabrication technologies are already optimized. A semiconductor platform allows for integration of quantum and classical networks.

\section{DISCUSSION}

\section{A. Figure of merit for split-ring polariton condensate qubits}

The simulations described above demonstrate that at certain conditions split-ring polariton condensates behave as two-level quantum systems demonstrating long-standing coherent oscillations, which are indicative of the continuous relative phase change between the basis states. Considering this system as a qubit, one should be able to estimate its figure of merit given by the number of logic operations that can be performed before the coherence between the two levels is lost [17]. For any two-level system, the most important factors affecting its figure of merit are the spontaneous relaxation of the excited state and the pure dephasing. These processes can be successfully accounted for with the use of the density matrix approach. The master equation for density matrix $\rho=|\psi\rangle\langle\psi|$ (see Appendix A for the definition of $|\psi\rangle$ ) of the two-level system reads

$$
i \hbar \frac{d \rho}{d t}=[\hat{H}, \rho]+\gamma_{r} \mathcal{L}\left[\sigma_{-}\right] \rho+\gamma_{d} \mathcal{L}\left[\sigma_{z}\right] \rho,
$$

where $\hat{H}$ is a split-ring qubit Hamiltonian whose eigenstates are $|0\rangle$ and $|1\rangle, \mathcal{L}[\hat{A}] \rho=\hat{A} \rho \hat{A}^{\dagger}-\frac{1}{2}\left(\hat{A}^{\dagger} \hat{A} \rho+\rho \hat{A}^{\dagger} \hat{A}\right)$ is a Lindblad superoperator and $\sigma_{-}=\sigma_{x}-i \sigma_{y}$ where $\sigma_{x, y, z}$ are Pauli matricies. While the first term in Eq. (4) governs the coherent dynamics, the last two describe the interaction with the environment. The second term stands for the excited state relaxation corresponding to the spontaneous flip of the qubit state from the upper to the lower energy level, which occurs with the rate $\gamma_{r}$. For the regime of dynamical balance between the gain from the $\mathrm{CW}$ pump and the single polariton dissipation rate, the relaxation of the upper state of the condensate as a whole does not occur. Also, the current of polaritons composed of electrically neutral excitons and photons is protected from coupling to the electromagnetic radiation, which is resonant to the transition connecting the basis states. This is also due to selection rules which forbid radiative transitions between polaritons formed by the same exciton state. At the same time, the relaxation assisted by the emission of acoustic phonon, which appears to be efficient for quantum dot [60] and superconducting qubits [61], is suppressed because of the mismatch between the size of the condensate (tens $\mu \mathrm{m}$ ) and the acoustic phonon wave length in the frequency range corresponding to the qubit energy gap (in the order of few $\mu \mathrm{m}$ for the GaAs quantum well). Also, the phonon propagation velocity is about several $\mu \mathrm{m} / \mathrm{ns}$, which makes the phonon field effectively static with respect to the fast dynamics of the condensate. Hence acoustic phonons cannot interact with the 
condensate state as a whole. Therefore, the effect of relaxation of the condensate as a whole can be neglected.

The last term in Eq. (4) describes the effect of pure dephasing for off-diagonal elements of the density matrix written in the basis of $|0\rangle$ and $|1\rangle$ states. The dephasing typically has two contributions, namely, from the relaxation of population and from the phase relaxation. The latter effect arises from stochastic fluctuations of the basis states energies characterized by the rate $\gamma_{d}$. For the case of no phase relaxation, the off-diagonal elements $\rho_{01}=\langle 0|\rho| 1\rangle$ and $\rho_{10}=\langle 1|\rho| 0\rangle$ decay on the dephasing time $T_{2}=2 T_{1}$ [62] which is connected to the population relaxation rate $\gamma_{r}=T_{1}^{-1}$. However, the contribution from the pure dephasing is typically dominating. The decoherence in polariton systems is caused primarily by the combined effect of the polariton pair-scattering and quantum fluctuations of the condensate density [63]. The latter are accounted for by the noise term in Eq. (1), which originates from the stimulated scattering of excitons from the reservoir to the polariton condensate. From the simulations performed, we extract the coherence time as a characteristic time of the temporal decay of the first-order coherence function $g^{(1)}$ of the condensate, shown in Fig. 4(c). For the set of parameters used in our simulations that correspond to a conventional GaAs-based microcavity, the superposition state retains its coherence for a remarkably long time. Fitting the envelope of the delay dependence of the first-order coherence function with the exponential function $g^{(1)}(\tau)=\exp \left(-\tau / \tau_{c}\right)$, we estimate the coherence time $\tau_{c}$ to be over 100 nanoseconds. This is much beyond the single polariton lifetime (as short as $6 \mathrm{ps}$ in our case) and even beyond the coherence time of the condensate as a whole, which may reach a few nanoseconds in optical traps [64]. With a further improvement of the microcavity samples quality and tuning the system parameters one can expect an extension of the coherence times up to the millisecond range [65].

Thus, estimating the single logic operation time by the period of the oscillations on the Bloch sphere that is of the order of $125 \mathrm{ps}$ in our case, we end up with a figure of merit of more than $10^{3}$, that matches those of best superconducting qubits [5].

This high figure of merit can be achieved in a split-ring condensate because it is localized on a spot that is much smaller than the coherence length in the polariton system and because the overall phase of the condensate that is subject to a fast decoherence is fully decoupled from the superfluid phase current dynamics which defines the trajectory of the considered quantum system on a Bloch sphere. It is also important that the oscillating regime is sustained by the dynamical balance between gain and losses. Therefore the energy relaxation of the condensate as a whole does not occur.

One can see polariton split-ring condensates demonstrate the properties of robust two-level systems and can be considered as qubits. In order to fully characterize the applicability of polariton qubits for quantum information processing, one should address the issues of setting the initial quantum state of a polariton qubit, coupling between different qubits, elementary logic operations, and read-out of the information from a set of polariton qubits. In the rest of the paper we develop the concept of quantum information processing with use of split-ring polariton qubits.

\section{B. Qubit state initialization and single-qubit rotations}

Setting a split-ring condensate into a given quantum state can be achieved with use of a resonant short pump pulse focused on a specific spot of the ring. A similar technique has been employed for setting the phase of polariton Rabi oscillations [66]. The state in which the qubit is excited can be controlled by tuning the depth of the pump step, while the overall phase of the condensate can be set with the use of a weak coherent pumping by a resonant laser pulse [67].

A control of the nonresonant pump allows for implementation of single qubit properties. In particular, the total intensity of the pump affects the value of the energy gap between the basis states as is discussed in Appendix B. Tuning of the splitting in the two-level system is crucial for the implementation of multiqubit operations as it allows addressing specific qubits from the quantum register. Alternatively, the same goal can be achieved by lifting the degeneracy between the states characterized by the opposite angular momenta, which is equivalent to the presence of the synthetic gauge field in accordance with the energy diagram shown in Fig. 1(d). An existence of the similar synthetic gauge field for exciton polaritons was recently demonstrated by applying the crossed electric and magnetic fields [32]. The static electric field polarizes excitons while the magnetic field causes an energy shift of the moving dipole-polarized excitons due to the magneto-Stark effect [68]. The value of the energy shift is proportional to the exciton (polariton) momentum. Therefore, this scheme is capable of synthesizing of the effective gauge potential for polaritons. In Appendix F, we discuss the specific realization of such a gauge field for a ring of dipole-polarized exciton polaritons in the presence of the radial electric field and normal to the cavity plane magnetic field. In particular, it is demonstrated that a single-qubit dynamics is governed by the following Hamiltonian written in the truncated two-level basis:

$$
\hat{H}=\frac{\Delta_{0}}{2} \sigma_{z}+\frac{\delta \omega}{2} \sigma_{x},
$$

where $\Delta_{0}$ is the energy of the basis states splitting, and $\delta \omega$ is the bias parameter corresponding to the gauge field-induced splitting between the circular polariton flows with opposite momenta.

The presence of the synthetic gauge field is crucial for implementing the quantum logic with split-ring qubits. Manipulation of the persistent current states' splitting parameter $\delta \omega$ paves the way for realization of the most of the single-qubit quantum gates. Here we demonstrate the performance of the operations of elementary rotation of the qubit state vector, also known as Pauli rotation gates.

As discussed in detail in Appendix F, we assume that the gauge field responsible for this splitting is controlled by the external voltage applied to the ring electrodes [Fig 7(a)]. Pauli $X$ and $Y$ gates (in what follows denoted as $\mathcal{R}_{x}$ and $\mathcal{R}_{y}$ ) require a resonant periodic driving of the qubit. This is achievable with the ac electric pulses, which generate a time-periodic radial electric field polarizing excitons in the cavity plane. The driving force applied resonantly to the qubit triggers the periodic beats of population between the basis states $|0\rangle$ and |1) that are similar to atomic Rabi oscillations. In the Bloch sphere representation it corresponds to the rotation about the axes lying in the equatorial plane. This important example 


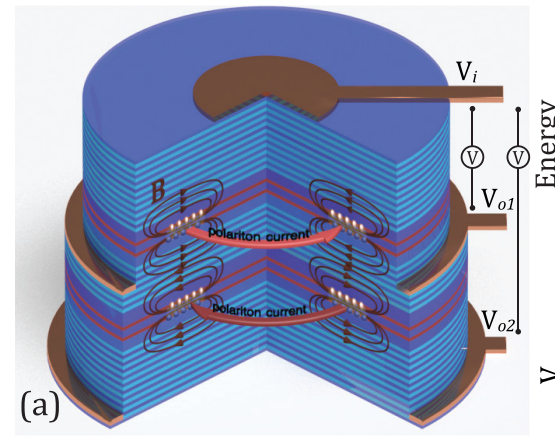

(a)

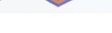

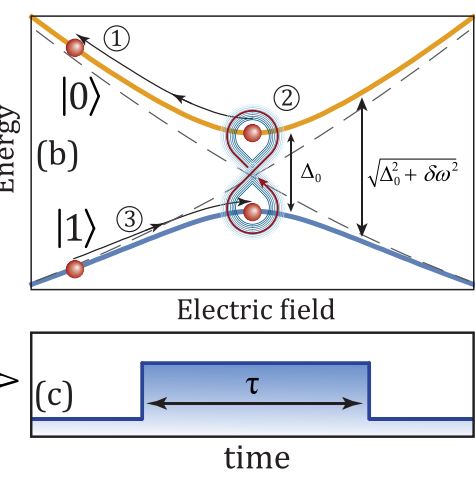

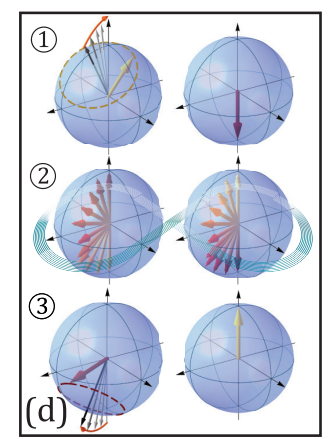

FIG. 7. Two-qubit processing with split-ring polariton condensates. (a) Schematic showing a double cavity with two coaxial dipolariton condensates. Different positions of stop bands of the two cavities allow for the selective nonresonant excitation and read-out of the qubits. Being detuned from the point of view of the polariton energy, two qubits are matched from the point of view of their energy gaps. Using the spatially indirect excitons formed in coupled quantum wells endows polaritons with the permanent dipole moment aligned perpendicular to the cavity plane. A single inner $\left(V_{i}\right)$ and a couple of outer $\left(V_{o 1}\right.$ and $\left.V_{o 2}\right)$ electrodes generate the radial electric field, which in combination with the perpendicular external magnetic field introduces an artificial gauge potential required for the selective tuning of the qubits' energy gap. (b) A three-step protocol for the implementation of the $i$ SWAP two-qubit gate. The energy diagram illustrates the manipulations with the energy gap of the first qubit. (c) The typical time dependence of the control electric field required for the realization of the iSWAP operation. (d) The schematic visualization of the Bloch vector behavior during the implementation of the iSWAP gate protocol. Being out of the resonance, the first qubit precesses about the effective magnetic field indicated be the gray arrow.

demonstrates that the radial electric field can serve as a versatile tool for coherent control of the quantum two-level system based on polariton split-ring condensate. As a drawback, a coupling to electromagnetic field opens an additional channel for the excited state relaxation via the radiative decay. However, in the regime of dynamic equilibrium, the upper state population should be continuously replenished by the pump.

The $\mathcal{R}_{x}$ and $\mathcal{R}_{y}$ rotations are distinguishable by the choice of the relative phase between the qubit and the driving force; see Appendix $G$ for details. Note that the ac driving of the qubit provides a convenient tool for the coherent control between the basis states. This technique can be used for the realization of Rabi or Ramsey interferometry experiments for verification of the predicted long-lived coherence of split-ring polariton qubits.

The $Z$-gate $\mathcal{R}_{z}(\theta)$ operation rotating the state vector by an angle $\theta$ about the $z$ axis can be efficiently replaced [69] with the addition of the time delay $\hbar \theta / \Delta_{0}$ between two successive single-qubit operations. Indeed, according to Hamiltonian (5) written in the frame of reference of the unbiased qubit, at $\delta \omega=0$, the state rotates with the frequency $\Delta_{0} / \hbar$ about the polar axis of the Bloch sphere. Such an equivalent of the Pauli $Z$-rotation operation should be properly addressed as a virtual or efficient $Z$ gate.

The Pauli rotations should be considered as the basis operations since their combinations can be used for the realization of new gates. In Appendix $G$ we demonstrate the synthesis of two successive unitary rotations resulting in implementation of the Hadamard gate, which allows for switching between the superposition and the basis states.

\section{Coupling between split-ring polariton qubits}

The interaction between split-ring condensates is a key ingredient for the realization of a scalable material platform for quantum computing. The efficient mechanism of the coherent coupling between polariton condensates is their exchange by polaritons propagating in the plane of a microcavity. This simple coupling scheme proved efficient for pairing of nearest neighbors in an array of polariton condensates employed in $X Y$ simulators [20]. In order to achieve coupling of distant condensates one can use 1D optical wave guides imprinted lithographically in the microcavity plane [70].

Here we propose an alternative approach that allows for fabrication of compact two-qubit gates. We demonstrate that the coupling between split-ring qubits is possible due to the magnetic field-mediated interaction between dipole-polarized condensates. If polaritons are polarized in the direction normal to the cavity plane, their circular flow induces separated counterpropagating electric currents comprising the carriers of opposite signs. The magnetic field generated by these currents affects another condensate excited nearby, which picks up the magnetic field in the same fashion as an inductive coil in an electric transformer. The interaction between the qubits, as expected, is maximized in the stacked geometry realized with the double cavity [Fig. 7(a)] made with use of the diluted magnetic semiconductors such as CdMnTe to increase the magnetic susceptibility of the system.

With the truncation to the two-level basis, the Hamiltonian of the magnetic field-mediated qubit-qubit interaction reads (see Appendix E)

$$
\hat{H}_{\mathrm{int}}=g \sigma_{x}^{(1)} \otimes \sigma_{x}^{(2)},
$$

where the Pauli $x$-operator $\sigma_{x}^{(\mathrm{i})}$ acts on $i$ th qubit spinor $\boldsymbol{\psi}^{(\mathrm{i})}=$ $(|0\rangle,|1\rangle)^{T} ; g$ is the interaction strength associated with the mutual inductance of the ring condensates of dipolar polaritons. Equation (6) resembles the Heisenberg Hamiltonian describing the exchange interaction between two spins. The interaction of this type is known for its efficiency for quantum computing [71,72]. In particular, it is naturally suitable for the state swapping operations as will be demonstrated in the next subsection.

Another important advantage of the inductive coupling is that its strength can be controlled by the external bias. This 
can be demonstrated recalling that in the diagonal basis the state of the individual qubit precess about the $z$-axis according to

$$
\hat{H}^{(\mathrm{i})}=\Delta_{i} \sigma_{z}^{(\mathrm{i})} / 2
$$

where $\Delta_{i}=\sqrt{\Delta_{0 i}^{2}+\delta \omega_{i}^{2}}$ is the $i$ th qubit eigenenergy in the presence of the bias. Therefore, in the rotating wave approximation, which implies neglecting the rapidly oscillating terms, the interaction Hamiltonian reads

$$
H_{\mathrm{int}}=g\left(e^{i \delta t / \hbar} \sigma_{+}^{(1)} \otimes \sigma_{-}^{(2)}+e^{-i \delta t / \hbar} \sigma_{-}^{(1)} \otimes \sigma_{+}^{(2)}\right),
$$

where $\sigma_{ \pm}=\sigma_{x} \pm i \sigma_{y}$ are the rising and the lowering operators which switch the qubit state between two poles; $\delta=\Delta_{1}-\Delta_{2}$ is an energy detuning between the qubits. At resonance, $\Delta_{1}=$ $\Delta_{2}$, the interaction (8) acts to exchange the quantum states between the qubits. The two-qubit state then oscillates with the frequency $2 g / \hbar$ between the states $|0\rangle \otimes|1\rangle$ and $i|1\rangle \otimes|0\rangle$; see Appendix H. On the other hand, out of resonance the interaction appears to be effectively suppressed due to the time averaging of the interaction Hamiltonian (8). This remarkable effect can be exploited for the realization of the on-demand switching of qubit-qubit interaction by tuning the corresponding eigenfrequencies with the electric field

\section{Realization of the $i$ SWAP gate with coupled split-ring polariton condensates}

As a practically important example of application of the inductive coupling of the split ring qubits, we propose the protocol of implementation of the $i$ SWAP two-qubit gate, as Fig. 7 illustrates. The $i$ SWAP operation is designed to permute the states of the two qubits with the addition of $\pi / 2$ phase difference. The protocol consists of three steps. In the first step, two qubits are prepared in a state characterized by the mismatch in their energy gaps. The energy mismatch guarantees that the magnetic field-mediated interaction is suppressed. Then we bring the qubits into resonance adiabatically tuning the pump intensity or using the external electric field; see Fig. 7(b). This triggers the recurring exchange of the states between the qubits at the frequency $2 g / \hbar$. The oscillations are interrupted after the time period $\tau=\hbar \pi / 2 g$ by detuning the qubits out of resonance. This constitutes the third step of the protocol. As a result of this gate operation, the condensates exchange their quantum states with the acquisition of the relative $\pi / 2$ phase shift.

\section{E. Qubit state read-out}

Finally, the read-out of a quantum state of the qubit can be done combining the time- and spatially resolved photoluminescence and interferometry measurements [56]. Note that this is a "weak measurement" method that does not fully destroy the measured quantum state, while it perturbs it to some extent. Conceptually, in a similar way, a SQUID-based read-out perturbs but does not fully destroy the quantum state of a superconducting flux qubit $[5,73]$. The proposed optical read-out technique is currently being used for studies of $X Y$ simulators based on an array of exciton-polariton condensates [20].

\section{F. Implementation of Deutsch's algorithm with coupled split-ring polariton condensates}

The use of an electric field for implementation of both single- and two-qubit gate operations allows for concatenation of these gates into the logic circuits suitable for realization of practical quantum algorithms. As an illustrative example of a high potentiality of the polariton split-ring qubit computational platform, we simulate here the implementation of the quantum Deutsch's algorithm [74].

Being one of the first oracle-based quantum protocols, Deutsch's algorithm was designed to demonstrate an advantage of quantum computing in a task of determining whether a coin is fair or fake. Clearly, in a classical domain, one needs at least two queries to cope with this task. The quantum Deutsch's algorithm, in contract, requires one examination step only.

The work of the algorithm can be illustrated using a set of four functions $f_{i}$, where $i \in\{1,2,3,4\}$, which map the input bit $x$ (being either 0 or 1 ) onto the output bit $y$, i.e., $y=f_{i}(x) \in$ $\{0,1\}$. The functions $f_{i}$ can be divided into two classes: the constant functions

$$
f_{1}(x)=0 \text { and } f_{2}(x)=1,
$$

whose output does not depend on the input state, and the balanced functions

$$
f_{3}(x)=x, \text { and } f_{4}(x)=\operatorname{NOT}(x),
$$

where NOT is the classical bit-flip operation. It is assumed that one gets some function $f_{i}$ with unknown uniformly distributed random $i \in\{1,2,3,4\}$ as a black box. For classical bits, it is possible to characterize the given function as constant or balanced by measuring its output twice: tacking consequently 0 and 1 states as the input. The quantum Deutsch's algorithm copes with this problem within only one query. It represents each of the functions $f_{i}$ by the corresponding two-qubit quantum gate $\mathcal{U}_{i}$ (see Table I in Appendix I), which plays the role of an oracle. Incorporating these gates into the logical circuit shown in Fig. 8(a), one can determine whether the function is balanced or constant by only a single call of the oracle. The answer to the problem appears to be encoded in the output state of the first qubit: The qubit is in the state $|1\rangle$ for balanced functions and in the state $|0\rangle$ in the opposite case.

The gates $\mathcal{U}_{i}$ required for realization of Deutsch's algorithm are represented by the identity and the bit-flip operations for the constant functions (9a) and by the controlled NOT-gate and the zero-controlled NOT-gate for balanced ones (9b). All these functions can be synthesized from the single-qubit Pauli rotations and the iSWAP gates; see Appendixes $\mathrm{H}$ and I for the gate identities.

For the practical realization of Deutsch's algorithm with the split-ring condensates one needs to prepare two polariton qubits designed with the mismatch of their energy gaps, say, $\Delta_{2}>\Delta_{1}$. The $i$ SWAP-gate has to be implemented by applying the DC voltage pulse on the first electrode (see Fig. 7), which governs the bias of the qubit no. 1 . The pulse amplitude is chosen such as to bring the first qubit into the resonance with the second, $\max \left[\delta \omega_{i \mathrm{SWAP}}\right]=\sqrt{\Delta_{2}^{2}-\Delta_{1}^{2}}$. The Hadamard 


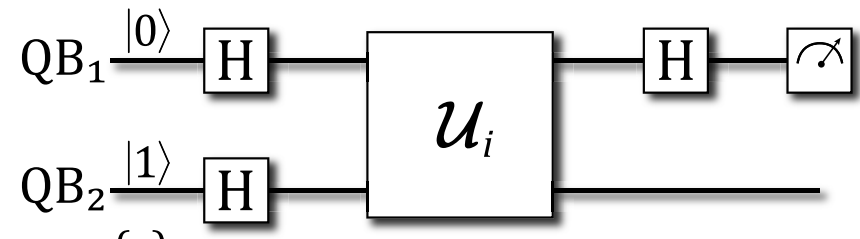

(a)

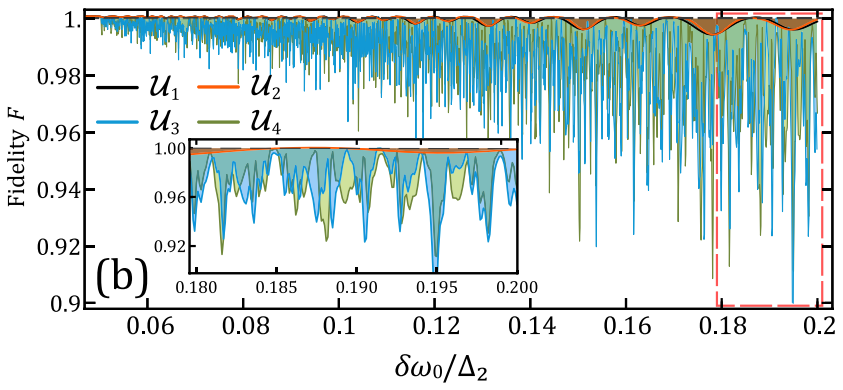

FIG. 8. Implementation of Deutsch's algorithm. (a) The logical circuit. $\mathbf{H}$ denotes the Hadamard transform. The output state of the first qubit is measured to detect whether the coin (function) is fair (balanced) or fake (constant). (b) The total fidelity of the algorithm simulated at different levels of the control pulse amplitude $\delta \omega_{0}=$ $\max \left[\delta \omega_{1}(\mathrm{t})\right]=\max \left[\delta \omega_{2}(\mathrm{t})\right]$. Four realizations of the algorithm corresponding to the oracles $\mathcal{U}_{1}, \mathcal{U}_{2}, \mathcal{U}_{3}, \mathcal{U}_{4}$ are indicated by black, orange, blue, and green, respectively. An example of the electric pulse sequence required for implementation of the $\mathcal{U}_{3}$ gate is illustrated in Appendix I.

gates are realized with two AC pulses as is described in Appendix G.

Figure 8 illustrates the total fidelity of the algorithm measured as $F=\left|\left\langle\left.\Psi\right|_{\text {out }, i} \mathcal{U}_{\mathrm{D} i} \mid \Psi\right\rangle_{\text {in }}\right|^{2}$, where $|\Psi\rangle$ is a fourcomponent vector of the two-qubit system with $|\Psi\rangle_{\text {in }}=|01\rangle$ being the input state and $|\Psi\rangle_{\text {out }, i}$ being the simulated output of the total algorithm realized with the gate $\mathcal{U}_{i} ; \mathcal{U}_{\mathrm{D} i}$ denote the transformation matrices of the ideal circuit with the gate $\mathcal{U}_{i}$. For the details of simulations see Appendix I. The main factor reducing the algorithm fidelity is the magnitude of the control pulse parametrized by the bias amplitude $\delta \omega_{0}=\max [\delta \omega(t)]$ in Fig. 8(b). Indeed, the accuracy of the Pauli rotation operations (specifically, $\mathcal{R}_{z}$ ) is limited by the validity of the rotating wave approximation, which typically assumes that the driving force is weak. For simple realizations such as those involving constant gates $\mathcal{U}_{1}$ and $\mathcal{U}_{2}$, the fidelity precesses close to $F=1$ as the ratio $\delta \omega_{0} / \Delta_{2}$ grows. The sequence of local rotations applied during the implementation of the more complicated realization of the quantum algorithm results in the frequent beating of the total fidelity; see the curves describing $\mathcal{U}_{3}$ and $\mathcal{U}_{4}$ quantum gates in Fig. 8. Notably, the average error always remains within a few percent. It justifies a high potential of the developed microcavity platform for quantum computing.

\section{CONCLUSIONS}

We have demonstrated that a coherent many-body quantum system represented by a bosonic condensate of excitonpolaritons placed in a split-ring geometry sustains stable and long-living oscillations between two circular current states. The polariton system qualitatively reproduces the behavior of a superconducting flux qubit. In a remarkable similarity to the flux qubit, in the considered split-ring polariton condensate a two-level quantum system is formed by superposition states of clockwise and anticlockwise circular currents. The size of the system is much less than the coherence length of a polariton condensate, which is why superfluid polariton currents are well preserved. This ensures a high figure of merit for qubits based on split-ring polariton condensates. We propose a specific approach for implementation of single- and two-qubit logic operations which implies application of the external electric field for the coherent control of the qubit state. Namely, we design an iSWAP two-qubit gate, which is known for its capability of producing the entangling gates required for a universal quantum computing. The present analysis paves the way to the realization of a new semiconductor platform for quantum information processing. The evident advantages of the considered quantum system are in its high scalability, high operation temperature, ultrafast logic operations, and potential integrability with classical semiconductor based nano-electronic devices.

\section{ACKNOWLEDGMENTS}

This work is supported by the Westlake University (Project No. 041020100118), Jilin University (the Fundamental Research Funds for the Central Universities), National Natural Science Foundation of China (Grant No. 12074147), and Program 2018R01002 funded by the Leading Innovative and Entrepreneur Team Introduction Program of Zhejiang. X.M. and S.S. acknowledge the Deutsche Forschungsgemeinschaft (DFG) through the TRR142 (Grant No. 231447078, project A04) and Heisenberg program (Grant No. 270619725). I.C. acknowledges funding from National Natural Science Foundation of China (Grant No. 12050410250). The support from RFBR grant 21-52-10005, from the Grant of the President of the Russian Federation for state support of young Russian scientists No. MK-4729.2021.1.2 and No. MK-5318.2021.1.2, and from the state task for VlSU in the scientific activity project 0635-2020-0013 are acknowledged. E.K. acknowledges a Russian Science Foundation Grant (19-71-10091) for supporting work on quantum algorithms (Sec. III F and Appendix I). The work of A.F. and A.K. was supported by Rosatom in the framework of the Roadmap for Quantum computing. A.K. acknowledges fruitful discussions with Boris Altshuler and Yuriy Rubo.

\section{APPENDIX A: MAPPING THE DYNAMICS ON THE BLOCH SPHERE AND CALCULATION OF THE MEAN ANGULAR MOMENTUM}

For a qubit based on a two-level system formed by the states $|0\rangle$ and $|1\rangle$, any state $|\psi\rangle$ on the surface of the Bloch sphere can be represented as a linear combination of two basis states: $|\psi\rangle=\alpha|0\rangle+\beta|1\rangle$, where the normalization condition $|\alpha|^{2}+|\beta|^{2}=1$ is implied. The circular current states can be represented as $|\circlearrowright\rangle=e^{-i \frac{\pi}{4}}(|0\rangle+i|1\rangle) / \sqrt{2},|\circlearrowleft\rangle=$ $e^{i \frac{\pi}{4}}(|0\rangle-i|1\rangle) / \sqrt{2}$. For simplicity, we associate $|m \simeq \pm 0.4\rangle$ [points corresponding to Figs. 3(b) and 3(d)] with $|\circlearrowleft\rangle$ and

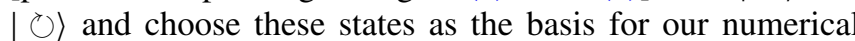


fitting procedure. In this way, we obtain

$$
|\psi\rangle=\alpha_{0}|m \simeq 0.4\rangle+\beta_{0}|m \simeq-0.4\rangle .
$$

The quantum state visited by the condensate can be characterized by a pseudo-spin vector $\hat{\mathbf{S}}=S_{x} \hat{\mathbf{e}}_{x}+S_{y} \hat{\mathbf{e}}_{y}+S_{z} \hat{\mathbf{e}}_{z}$ whose components are defined as $S_{x}=\frac{1}{2}\left(\alpha \beta^{*}+\alpha^{*} \beta\right), S_{y}=$ $\frac{i}{2}\left(\alpha^{*} \beta-\alpha \beta^{*}\right), S_{z}=\frac{1}{2}\left(|\beta|^{2}-|\alpha|^{2}\right)$. The mapping of the condensate dynamics to the Bloch sphere is realized using the method of maximum inherit optimization.

We note that the current direction at the position of the slot is different for the inner part $\left(r<r_{0}\right)$ and the outer part $(r>$ $r_{0}$ ) of the polariton condensate, as shown in the right panels of Figs. 3(b)-3(d) $\left(r_{0}=8 \mu \mathrm{m}\right)$. Figure 3(a) shows the angular momentum for the outer part of the polariton condensate that manifests a pronounced superfluid phase current.

\section{APPENDIX B: ENGINEERING ENERGY GAP WITH THE C-SHAPE OPTICAL PUMP}

Although the C-shape pump shown in Fig. 2 has a complicated profile, it is the radial slot which plays the most essential role. It governs the energy splitting of the basis states, which is a key characteristic of the qubit. In this section we consider how the incoherent pump can be used to control the value of the energy gap. Properties of the basis states $|0\rangle$ and $|1\rangle$ are analyzed with the simplified $1 \mathrm{D}$ equivalent of the $2 \mathrm{D}$ model (1). This assumption is relevant to the limit of a thin ring with a large mean radius $R_{0}$ [55]. The transformation to the $1 \mathrm{D}$ model is performed with a substitution $\psi(\mathbf{r}, t)=$ $\Phi(\rho) \bar{\psi}(\phi, t)$ and $n_{R}(\mathbf{r}, t)=N_{R}(\rho) \bar{n}_{R}(\phi, t)$, where $\Phi(\rho)$ and $N_{R}(\rho)$ account for the radial distribution of the condensate and the reservoir, respectively, $\rho$ and $\phi$ are polar coordinates. After integrating out the radial dependence and neglecting the stochastic term, one reduces Eqs. (1) to the two coupled 1D ordinary differential equations for $\bar{\psi}(\phi, t)$ and $\bar{n}_{R}(\phi, t)$ with $\nabla^{2} \rightarrow R_{0}^{-2} \partial_{\phi \phi}$.

Then tacking $\partial \bar{n}_{R} / \partial t=0$ and $\bar{\psi}(\phi, t)=\bar{\psi}_{0}(\phi) \exp (-i \mu t)$ we solve the obtained stationary problem for $\bar{\psi}_{0}(\phi)$ and $\bar{n}_{R}(\phi)$ iteratively using the Newton-Raphson algorithm. This method yields the azimuthal dependence of the wave function and the corresponding eigenenergy $\hbar \mu$ simultaneously. The typical shapes of the basis state wave functions are displayed in Figs. 2(c) and 2(d). Note that the solutions predicted with the model of the reduced dimensionality demonstrate a good agreement with those shown in Fig. 6, which were obtained from the dynamical simulations performed using the full 2D model. It justifies the validity of the used approach.

The dependencies of the energy splitting between the states $|0\rangle$ and $|1\rangle$ on the pump parameters are shown in Fig. 9. We focus on the impact of the slot width $w_{d}$ and the top-hat region amplitude $P_{0}$. For simplicity, we do not account for the pump step, working with the pump profile illustrated in Fig. 2(b). Note that the gap $\Delta_{0}$ demonstrates a nonmonotonic behavior as a function of the total pump power; see Fig. 9(a). Although the particular $\Delta_{0}(P)$-behavior essentially depends on the slot width, the gap typically decreases close to the condensation threshold $P^{\text {th }}$ and saturates for the pump power about several times the threshold. The complicated dependence of the energy gap on the pump intensity indicates a significant role of the polariton-polariton interactions, which are responsible
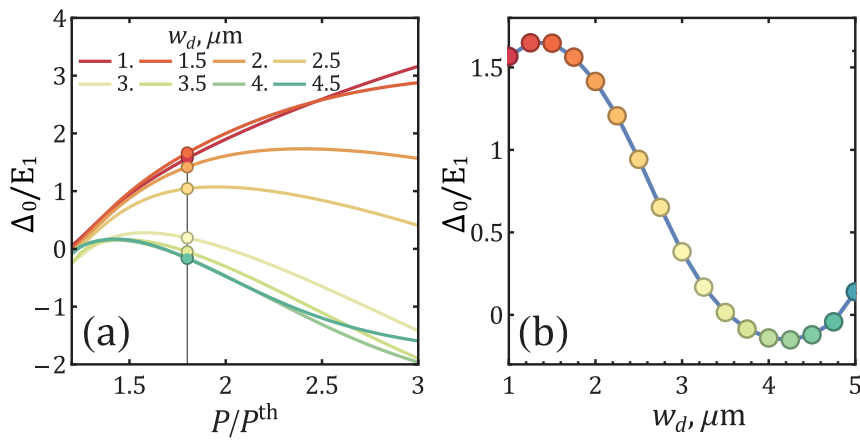

FIG. 9. Properties of the energy gap $\Delta_{0}$ between the qubit basis states measured in the units of energy of the circular current with a unit vorticity $E_{1}=\hbar^{2} / 2 m^{*} R_{0}^{2}$. The mean radius of the pump ring is $R_{0}=15 \mu \mathrm{m}$. Other parameters necessary for calculations are inherited from the full model (1).

for the dependence of the energy of the condensate on its population. The variation of the slot width allows for a fine tuning of the energy gap as is demonstrated in Fig. 9(b). For the considered parameters, the optimal value of the gap is achieved with the slot width being about a few micrometers and the pump power being not too far above the threshold.

Since the pump power strongly affects the energy splitting, the pumping source should possess a high degree of the power stability. The intensity noise causes stochastic fluctuations of the qubit energy which imply effective dephasing and lead to the reduction of the coherence time. Therefore, the pump profile has to be designed tacking into account minimization of the slope of the $\Delta_{0}(P)$-dependence. On the other hand, a high sensitivity of the splitting upon the pump properties can be employed for tuning the qubit energy dynamically during realization of the single-qubit quantum operations. However, in this case the manipulation speed appears to be limited by the response time of a spatial light modulator used for the pump shape sculpturing. An alternative approach which implies the use of the synthetic gauge field, as discussed in Appendix F, appears to be suitable for manipulating the qubit basis states coherently. As well, this method is characterized by a fast response, which is governed by the timescale of the exciton dipole-polarization formation. Therefore, it is more promising for quantum computing applications.

\section{APPENDIX C: HALF-QUANTUM CURRENTS TRAPPED IN C-SHAPE POTENTIALS}

Circular superfluid currents with fractional angular momenta demonstrating persistent coherent oscillations can also be found in the case of a condensate confined to a C-shape external potential created, e.g., by etching of a planar microcavity sample. In order to describe the system in this case we introduce the additional stationary potential $V(\mathbf{r})$; see Eq. (1). We consider the nonresonant excitation of the system by a broad pump as illustrated in Fig. 10. The considered potential contains a narrow barrier, which is different from the optically induced potential distribution shown in Fig. 2. In the main text, the slot in the pump-ring corresponds to a potential well for the polariton condensate. Under the excitation by a nonresonant broad pump with a relatively low 

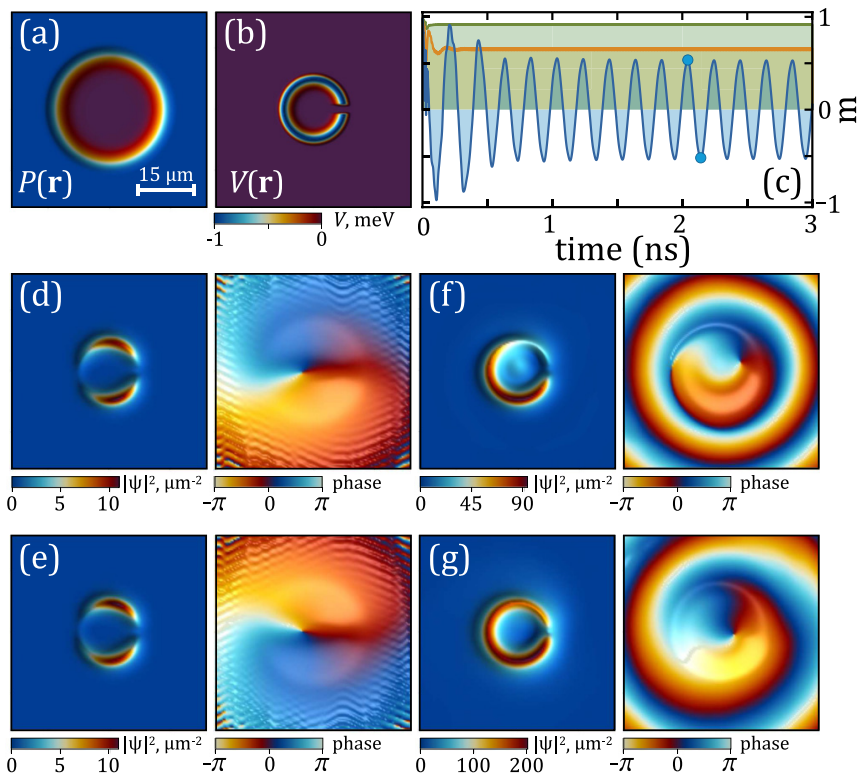

FIG. 10. Circular superfluid currents confined to a narrow Cshape external potential. (a) Pump profile with a flat top. (b) Narrow C-shape potential well with the potential depth of $1 \mathrm{meV}$. (c) Time evolution of the angular momentum of the condensate for different pump intensities: $P_{0}=1.3 \times P_{\text {th }}$ (blue line), $P_{0}=3.3 \times P_{\text {th }}$ (orange line), and $P_{0}=6.6 \times P_{\text {th }}$ (green line). Density (middle row) and phase (bottom row) distributions of the condensate at different pump intensities: (d), (e) $P_{0}=1.3 \times P_{\text {th }}$, (f) $P_{0}=3.3 \times P_{\text {th }}$, and (g) $P_{0}=$ $6.6 \times P_{\text {th }}$. Panels (d) and (e) correspond to the blue points in (c). $P_{\text {th }}$ is the condensation threshold. Here the parameters are $\gamma_{\mathrm{c}}=0.3 \mathrm{ps}^{-1}$, $m^{*}=2 \times 10^{-5} m_{\mathrm{e}}, g_{c}=3 \times 10^{-3} \mathrm{meV} \mu \mathrm{m}^{2}$.

intensity $\left(P_{0}=1.3 \times P_{\text {th }}\right)$, an oscillating state with its angular momentum varying between $m \simeq \pm 0.5$ is obtained, as shown in Figs. 10(c)-10(e).

The tunneling of polaritons through the narrow barrier mimics the Josephson dynamics. It is important to underline that Josephson oscillations between two condensates observed in Ref. [75] decay on the timescale of the coherence time of polariton condensates because of the decoherence between two condensates. In contrast, in the ring geometry we work with a single condensate. The fluctuations of its overall phase do not affect the phase difference between its parts situated to the right and left sides of the potential barrier, which is why the oscillations persist on a much longer timescale in our case. We estimate that the decoherence time in our system scales exponentially with the ratio of the coherence length to the diameter of the ring condensate. It may exceed several tens of nanoseconds in realistic GaAs-based microcavities.

As the pump intensity increases, the system achieves a steady-state regime where the normalized angular momentum is fractional, as shown in Fig. 10(f). Note that this state is different from those in Figs. 3(b) and 3(d), since there is no clear $\pi$ phase jump at the potential barrier here. Making the pump intensity much stronger, one can see clearly the tunneling of polaritons under the potential barrier; see Fig. $10(\mathrm{~g})$. The tunneling ensures a smooth phase variation in this case, so that the angular momentum of the condensate approaches $m=1$; see Fig. 10(c).
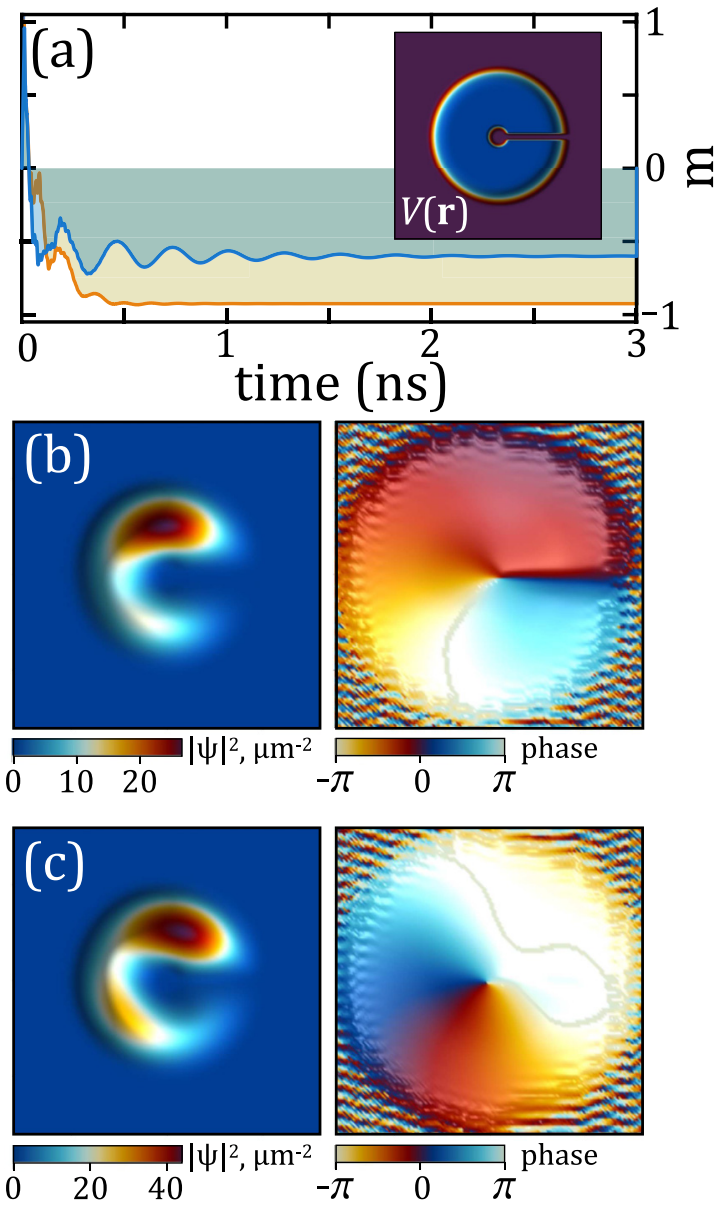

FIG. 11. Circular superfluid currents confined to a wide C-shape external potential. (a) Time evolution of the angular momentum for different pump intensities: $P_{0}=2 \times P_{\text {th }}$ (blue) and $P_{0}=2.6 \times P_{\text {th }}$ (orange). The inset shows the broad C-shape potential well of the depth of $1 \mathrm{meV}$. Density (right column) and phase (left column) distributions of condensates at different pump intensities: (b) $P_{0}=$ $2 \times P_{\text {th }}$ and (c) $P_{0}=2.6 \times P_{\text {th }}$.

We note that the shape of the external potential strongly influences the spatial distribution of the polariton density in the condensate. If the potential width is increased [inset to Fig. 11(a)], the same broad pump as in Fig. 10(a) can create a C-shape solution with the angular momentum $m \simeq \pm 0.5$ [Fig. 11(a)], and a clear $\pi$ phase jump [43] is observed at the potential barrier as shown in Fig. 11(b). This solution is very similar to that of Fig. 3. As in the previous case, while the pump intensity increases, the phase difference between both sides of the potential barrier changes from $\pi$ to 0 , leading to the formation of the current state with an integer angular momentum; see Figs. 11(a) and 11(c).

\section{APPENDIX D: SPECTRAL ANALYSIS OF THE OSCILLATIONS IN A SPLIT-RING POLARITON CONDENSATE}

The strong evidence of the two-level nature of the oscillating split-ring polariton condensate is provided by its energy spectrum. The Fourier spectrum of the dynamics of the condensate wave function (see Fig. 3) $S(\omega)=$ 


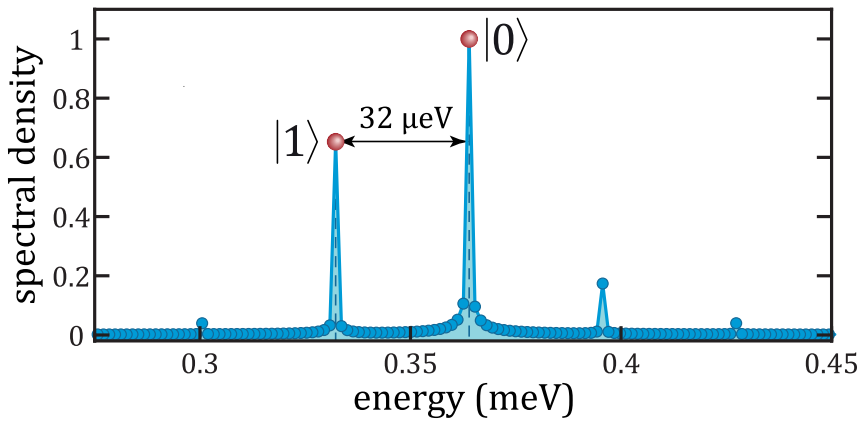

FIG. 12. The energy spectrum of the split-ring condensate in the oscillating regime corresponding to Fig. 3.

$\iint \psi(t, \mathbf{r}) e^{-i \omega t} d t d \mathbf{r}$ is shown in Fig. 12. Note that besides the pair of peaks corresponding to the eigenstates of the system $|0\rangle$ and $|1\rangle$ shown in Fig. 6, the comb of the side-band peaks of attenuated intensities also appears in agreement with recent predictions [76,77]. These satellites result from the nonlinear processes in the driven-dissipative polariton system and are indicative of the deviation of the considered split-ring condensate from an ideal two-level linear quantum system. The intensities of the side peaks increase as the pump power increases and the interactions between counter-rotating polariton currents become important.

\section{APPENDIX E: MAGNETIC FIELD-ASSISTED COUPLING BETWEEN CIRCULAR POLARITON CURRENTS}

Polaritons are composed of the electrically neutral excitons and photons. Therefore, in the first-order approximation over the ratio on the exciton Bohr radius to the magnetic length their motion is typically uncoupled from the magnetic field in the sense that moving polaritons experience the same energy shift from the magnetic field as the polaritons at rest. In particular, it is true for the polaritons formed by the ground-state excitons, which possess no electric dipole moment. However, it is not correct for those polaritons, which appear due to the strong coupling between the light and the dipole-polarized excitons. The stationary dipole moment is characteristic for excitons excited, e.g., in coupled asymmetric quantum wells, where the electron and the hole are localized in spatially separated layers. This particular case is illustrated in Fig. 7. Also, the exciton dipole moment can be induced and, what is more important, controlled by applying an external electric field. The latter case has a crucial importance for tuning the qubit energy gap, as we show in the next section.

Now let us consider a ring-shaped condensate of dipolepolarized polaritons. In what follows we address the properties of the excitonic component only and neglect the spin-dependent effects. However, since the light-matter interaction in the strong coupling regime implies conservation of momentum, the exciton and the polariton momenta are identical. Therefore, the coupling of the dipolar exciton motion with the magnetic field is naturally imprinted on polariton properties with the scaling factor given by the squared excitonic Hopfield coefficient $C_{x}$.

According to Ref. [78] the motion of the exciton center of mass is coupled to its internal structure in the presence of the external magnetic field $\mathbf{B}$. This coupling is reflected in the appearance of the extra term in the excitonic Hamiltonian:

$$
\hat{H}_{\mathrm{ex}} \propto \frac{e[\mathbf{d} \times \mathbf{B}]}{M_{\mathrm{ex}}}(-i \hbar \nabla),
$$

where $e$ is the elementary charge, and $M_{\mathrm{ex}}$ is the exciton effective mass associated with its motion as a whole particle. The bold symbols are used for the vectorial variables. In particular, $\hbar \mathbf{k}=-i \hbar \nabla$ is the exciton momentum, and $e \mathbf{d}$ is the electric dipole moment. The Hamiltonian (E1) implies that the coupling is maximized provided that the electric dipole, magnetic field, and exciton momentum are mutually orthogonal. Therefore, for the polaritons polarized perpendicularly to the microcavity plane, $\mathbf{d}=\mathbf{d}_{z}$, and rotating about the $z$ axis, $\mathbf{k}=\mathbf{k}_{\varphi}$, it is the radial component $\mathbf{B}_{\rho}$ of the magnetic field that affects the polariton motion. Here $z, \rho$, and $\varphi$ are the cylindrical axes. In this particular configuration the radial magnetic field modifies the exciton dispersion shifting its minimum out of the zero-momentum state.

The effect, which is in some sense reciprocal to the one described above, is the generation of the magnetic field by a ring of rotating dipolar exciton polaritons. Indeed, the motion of the exciton is equivalent to the two counterpropagating currents of the oppositely charged electrons and holes. Since the carriers are separated in space, the magnetic fields they generate do not compensate each other. The resultant field is maximized in the interlayer region where the radial component $\mathbf{B}_{\rho}$ is the dominating one. For the magnetic field generated by the polariton fluid with the unit vorticity (whose winding number is one), its out-of-plane radial component is

$$
B_{\rho}=\frac{\mu_{0} e \hbar C_{x}^{2} n_{\mathrm{pol}}}{2 M_{\mathrm{ex}} \rho^{2}} d,
$$

where $n_{\mathrm{pol}}$ is the polariton density, $C_{x}^{2}$ is the squared exciton Hopfield coefficient that quantifies a contribution from the excitonic fraction. Note that the expression (E2) is valid in the region where $\rho \gg h \gg d$, where $h$ is the distance from the quantum well plane. The more general expression can be found in Ref. [79].

According to the previous discussion, the field (E2) generates the polariton current in the coaxial polariton condensate excited in the adjacent microcavity located at the distance $h$. This effect can be taken into account in the driven-dissipative Gross-Pitaevskii equation (1). The Hamiltonian (E1) yields additional k-dependent terms in the relevant Schrödinger equation for the exciton wave function. They can be accounted for in Eq. (1) using the coupled oscillator model. Here we omit the rigorous derivation of the relevant expression leaving it for a more detailed study (see also Ref. [32]).

Also, for the sake of simplicity we consider the limiting case of the homogeneous polariton density disregarding the dark soliton perturbations in the vicinity of the defect. With this approximation (1) for the wave function of the first condensate $\Psi^{(1)}$ acquires an additional term which describes a coupling with the second condensate associated with the wave function $\Psi^{(2)}$.

$$
i \hbar \frac{\partial \Psi^{(1)}}{\partial t} \propto \frac{\mu_{0} e^{2} d^{2} \hbar}{2 M_{\mathrm{ex}}^{2} R_{0}^{3}} C_{x 1}^{2} C_{x 2}^{2} n_{\mathrm{pol}}^{(2)} \hat{L}_{z} \Psi^{(1)},
$$


where we used a nonstochastic form of Eq. (1) and skipped all terms but one which arises from the Hamiltonian (E1) with the use of (E2). The values $C_{x 1}$ and $C_{x 2}$ are assigned to the excitonic Hopfield coefficients characterizing the first and the second condensates, correspondingly. In Eq. (E3) we defined the angular momentum operator as $\hat{L}_{z}=-i \hbar \partial / \partial \varphi$. This assumption is valid for the quasi-1D case, i.e., for the condensate localized on a thin ring with the radius $R_{0}$. Then, defining the average angular momentum per unit area of the second condensate as $L_{z}^{(2)}=S^{-1} \int_{S}\left(\Psi^{(2)}\right)^{*} \hat{L}_{z} \Psi^{(2)} d \mathbf{r}=\hbar m n_{\text {pol }}^{(2)}$, where $m$ is a mean angular momentum defined in the main text, we obtain

$$
i \hbar \frac{\partial \Psi^{(1)}}{\partial t} \propto g L_{z}^{(2)} \hat{L}_{z} \Psi^{(1)},
$$

where $g=\frac{\mu_{0} e^{2} d^{2}}{2 M_{\mathrm{ex}}^{2} R_{0}^{3}} C_{x 1}^{2} C_{x 2}^{2}$.

Associating the condensate angular momentum with the $x$ component of the pseudospin state $\psi$ written in the basis of the nonrotating states $|0\rangle$ and $|1\rangle$, we can write the truncated Hamiltonian which is capable of describing the evolution of the coupled split-ring qubits:

$$
\hat{H}_{q q}=\sum_{\mathrm{i}=1,2} \Delta_{i} \sigma_{z}^{(\mathrm{i})} / 2+g \sigma_{x}^{(1)} \otimes \sigma_{x}^{(2)},
$$

where $\Delta_{i}$ defines the energy gap between the basis states of the $i$ th qubit and the $\otimes$ sign emphasizes the tensor product. Note that the coupling strength $g$ can be associated with the effective mutual inductance using the analogy with the superconducting flux qubit systems.

\section{APPENDIX F: TUNING THE ENERGY GAP WITH THE ARTIFICIAL GAUGE FIELD}

Implementation of the most of the two-qubit quantum gates implies a sequence of operations with the individual qubits. In particular, for the realization of the $i$ SWAP gate protocol proposed in the main text, one needs a tool for tuning the energy gap between the qubit basis states. In the case of superconducting flux qubits, this is easily achievable by varying the external magnetic flux [see Fig. 1(c)]. For the exciton polaritons such a strategy is also possible. However, it requires the synthetic gauge field which would act on the electrically neutral polaritons. As was demonstrated in Ref. [32], such an artificial field arises from the coupling between the motion of a polarized exciton and the magnetic field given by Eq. (E1). Here we explain how this approach can be used for tuning of the energy gaps in the split-ring polariton condensates.

We consider the setup shown in Fig. 7(a). An external electric field $\mathbf{E}$ has a radial component which induces the exciton polarization characterized by the dipole moment $\mathbf{d}_{\rho}=\alpha \mathbf{E}_{\rho}$, where $\alpha$ is the exciton polarizability. The external magnetic field is uniform, and it is directed perpendicularly to the microcavity plane, $\mathbf{B}=\mathbf{B}_{z}$. This configuration guarantees the mutual orthogonality of the magnetic field, the dipole moment, and the azimuthal polariton momentum $\hbar \mathbf{k}_{\varphi}$. In this case, the artificial gauge potential $\mathbf{A}$, which defines the pulse rescaling rule $\hat{\mathbf{p}} \rightarrow \hat{\mathbf{p}}-\mathbf{A}$, reads

$$
\mathbf{A}=\mathbf{A}_{\varphi}=e\left[\mathbf{d}_{\rho} \times \mathbf{B}_{z}\right] C_{x}^{2} m^{*} / M_{\mathrm{ex}},
$$

where $m^{*}$ is the effective mass of polaritons on the lower dispersion branch. In the uniform polariton density approxi- mation that was assumed in the previous section and in the defect-free case the splitting between the states whose winding numbers differ by 1 is

$$
\delta \omega\left(E_{\rho}\right)=\frac{2 e \hbar C_{x}^{2} B_{z}}{R_{0} M_{\mathrm{ex}}} d_{\rho}\left(E_{\rho}\right) .
$$

Equation (F2) explicitly shows the electric field dependence of the energy bias parameter $\delta \omega$.

Note that the action of the effective field is equivalent to the condensate rotation about the $z$-axis. It lifts the energy degeneracy between the clockwise and anticlockwise currents. Therefore, in the truncated basis this rotation is equivalent to the appearance of the additional term in the single-qubit Hamiltonian:

$$
\hat{H}_{q}=\frac{\Delta_{0}}{2} \sigma_{z}+\frac{\delta \omega}{2} \sigma_{x},
$$

where $\Delta_{0}$ is the minimal value of splitting; see Fig. 7(b). Diagonalization of this Hamiltonian yields the energy gap between the qubit eigenstates:

$$
\Delta=\sqrt{\Delta_{0}^{2}+\delta \omega^{2}} .
$$

Note that at $\delta \omega \neq 0$ the qubit state precesses about the axis tilted with respect to the main axis of the Bloch sphere; see Fig. 7(d).

Expressions (F2) and (F4) quantify the relation between the qubit splitting and the electric field paving the way for the realization of single- and multi-qubit operations with splitring polariton condensates controlled by the time-dependent external bias. The protocols for their implementation are described in detail in Appendixes $\mathrm{G}$ and $\mathrm{H}$.

\section{APPENDIX G: SINGLE-QUBIT OPERATIONS}

Let us assume that the split-ring qubit is affected by the AC pulse of the radial electric field with the carrier frequency $\omega_{d}$. It periodically perturbs the persistent currents states whose energies are biased according to

$$
\delta \omega(t)=s(t) \cos \left(\omega_{d} t+\phi\right),
$$

where $s(t)$ accounts for the pulse envelope and $\phi$ stands for the relative phase between the driving signal and the qubit.

The effect of the driving can be clearly seen in the frame rotating with the qubit eigenfrequency. To transfer to this frame, we perform the unitary transformation $U_{R}=$ $\exp \left(-i \Delta \sigma_{z} t / 2 \hbar\right)$, which transforms the Hamiltonian (5) to $\hat{H}_{\mathrm{rf}}=U_{R} \hat{H} U_{R}^{\dagger}+i \dot{U}_{R} U_{R}^{\dagger}$. After dropping the fast rotating terms we are left with the following single-qubit Hamiltonian written in the rotating wave approximation (RWA):

$$
\begin{aligned}
\hat{H}_{\mathrm{RWA}}= & \frac{s(t)}{2}\left(\begin{array}{cc}
0 & e^{i\left(\omega_{d}-\Delta_{0} / \hbar\right) t+i \phi} \\
e^{-i\left(\omega_{d}-\Delta_{0} / \hbar\right) t-i \phi} & 0
\end{array}\right) \\
= & \frac{s(t)}{2}\left\{\cos \left[\left(\omega_{d}-\Delta_{0} / \hbar\right) t+\phi\right] \sigma_{x}\right. \\
& \left.-\sin \left[\left(\omega_{d}-\Delta_{0} / \hbar\right) t+\phi\right] \sigma_{y}\right\} .
\end{aligned}
$$

At the resonance $\omega_{d}=\Delta_{0} / \hbar$, the $\mathcal{R}_{x}$ - and $\mathcal{R}_{y}$-rotations can be distinguished by the appropriate choice of the relative phase $\phi$. In particular, at $\phi=0$ the qubit rotates about the $x$-axis, while an out-of-phase pulse $\phi=\pi / 2$ corresponds to the rotation about the $y$-axis. The rotation angle $\theta$ is determined by the pulse envelope: $\theta=\hbar^{-1} \int s(t) d t$. 

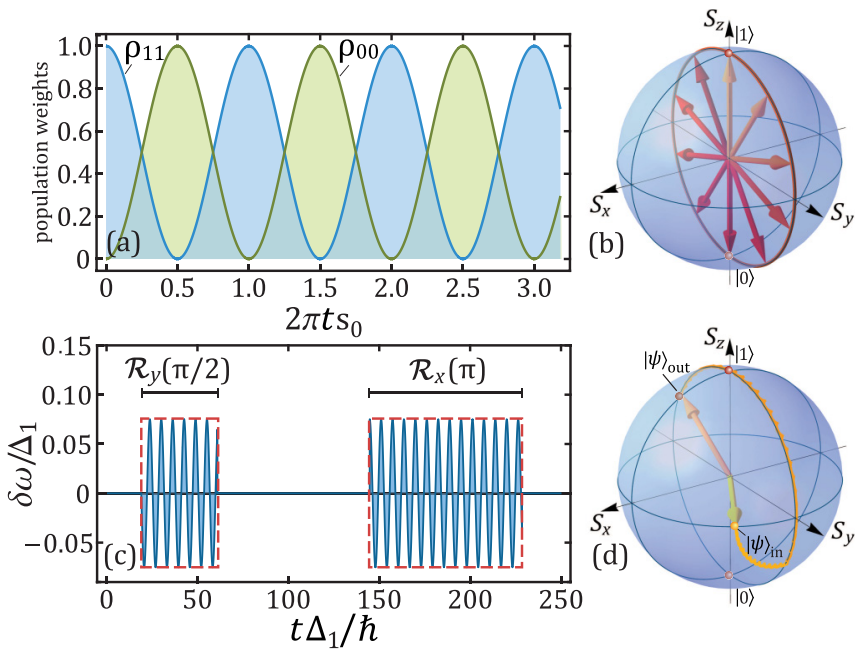

FIG. 13. (a) Rabi oscillations of the qubit basis states populations quantified as $\rho_{11}=|\langle 1 \mid \psi\rangle|^{2}$ and $\rho_{00}=|\langle 0 \mid \psi\rangle|^{2}$. (b) The relevant dynamics on the Bloch sphere. (c) Realization of the Hadamard gate composed of two successive Pauli rotations driven by the AC electric pulses. The envelope functions are taken to be rectangular. (d) Dynamics of the Bloch vector. The initial state taken as $|\psi\rangle_{\text {in }}=$ $\left(|0\rangle+e^{i \pi / 3}|1\rangle\right) / \sqrt{2}$ is shown with the yellow arrow, while the output state $|\psi\rangle_{\text {in }}$ corresponds to the red arrow.

It is instructive to consider a limiting case of a constant amplitude $s(t)=s_{0}$ which corresponds to the Rabi problem of a two-level system excited with the resonant periodic driving. Tacking $\phi=0$ leaves us with $\hat{H}_{\mathrm{RWA}}=s_{0} \sigma_{x} / 2$. Thus, the qubit excited in the state $|1\rangle$ starts a coherent recurrent transfer of populations between the upper and lower energy levels with the frequency $s_{0} / \hbar$ once the external electric field is applied. In the Bloch sphere representation, it corresponds to the periodic rotation of the qubit state about the $x$-axis; see Figs. 13(a) and $13(b)$.

In Sec. III B the Z-gate was shown to be equivalent to the introduction of time delay between two successive single-qubit operations. However, in practice, especially with many-qubit protocols, this approach fails when one needs to apply a local $\mathcal{R}_{z}$-gate to the given qubit remaining the other unaffected. The time interval between the local gates adds a phase to all the qubits in the register, since the time is running equally for all of them. The alternative approach is to shift the phase of the given qubit with respect to the others by manipulation of the energy gap of the target qubit. At finite $\delta \omega$ the precession axis of the qubit state tilts towards the $x$ axis while the frequency of precession increases to $\Delta / \hbar$ according to (F4). The qubit vector precesses faster and accumulates an additional phase shift. Therefore, applying the DC electric pulse on the given qubit, one shifts its relative phase on $\theta(t)=\hbar^{-1} \int_{t_{0}^{\prime}}^{t_{0}^{\prime}+t}\left(\sqrt{\Delta_{0}^{2}+\delta \omega\left(t^{\prime}\right)^{2}}-\Delta_{0}\right) d t^{\prime}$, which is equivalent to the additional $z$-rotation of the qubit state with respect to the unbiased case.

As was mentioned in the main part of the text, the Pauli rotations can be used for engineering other gates. The practically important example of a gate synthesis is a Hadamard operation, which performs a $\pi$ rotation about the axis diagonal in the $x$-z-plane. In particular, it translates the superposition state

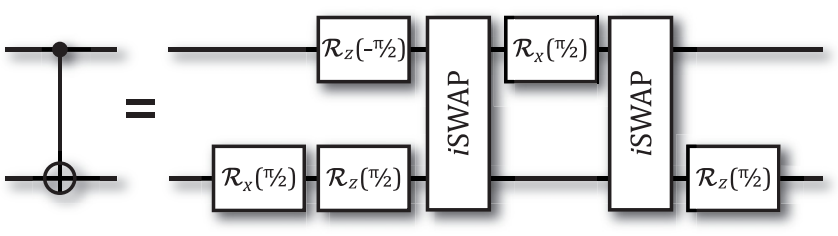

FIG. 14. Realization of the CNOT-gate with two iSWAPs and several local gates.

$(|0\rangle+|1\rangle) / \sqrt{2}$ to the pole of the Bloch sphere. The Hadamard gate can be generated by the sequence of two unitary rotations, namely, the $\pi$-rotation about the $x$-axis followed by the $\pi / 2$ rotation about the $y$-axis.

The scenario of implementation of the Hadamard operation with the approach proposed in this section is shown in Figs. 13(c) and 13(d). The shapes of electric pulses responsible for $\mathcal{R}_{x}$ and $\mathcal{R}_{y}$ rotations are shown on the left [Fig. 13(c)]. The Bloch vector dynamics is demonstrated in Fig. 13(d) with the yellow line on the sphere surface. The weak oscillations of the qubit trajectory appear due to the presence of the fast rotating terms in the total Hamiltonian (5), which were omitted in the rotating wave approximation (G2). The impact of this terms grows as the electric field amplitude increases.

\section{APPENDIX H: THE $i$ SWAP GATE}

The $i$ SWAP gate acts to swap the states between the qubits with the addition of $\pi / 2$ phase difference. Typically, this gate requires the presence of the $X Y$ qubit-qubit interactions [72]. Fortunately, this is the kind of coupling which is realized in the double-cavity system shown in Fig. 7; see Hamiltonian (E5). Indeed, in the rotating wave approximation, the evolution operator corresponding to the coupling term reads [80]

$$
\begin{aligned}
\mathcal{U}_{\text {int }} & =e^{-i \frac{g}{2 \hbar}\left(\sigma_{x} \sigma_{x}+\sigma_{y} \sigma_{y}\right)} \\
& =\left(\begin{array}{cccc}
1 & 0 & 0 & 0 \\
0 & \cos \left(\frac{g}{\hbar} t\right) & -i \sin \left(\frac{g}{\hbar} t\right) & 0 \\
0 & -i \sin \left(\frac{g}{\hbar} t\right) & \cos \left(\frac{g}{\hbar} t\right) & 0 \\
0 & 0 & 0 & 1
\end{array}\right),
\end{aligned}
$$

where we assumed that the qubits are in resonance, $\Delta_{1}=\Delta_{2}$. As is evident from (H1), at $t=\hbar \pi / 2 g$ the evolution matrix is identical to the $i$ SWAP operation, namely:

$$
\mathcal{U}_{i \mathrm{SWAP}}=\left(\begin{array}{cccc}
1 & 0 & 0 & 0 \\
0 & 0 & -i & 0 \\
0 & -i & 0 & 0 \\
0 & 0 & 0 & 1
\end{array}\right)
$$

Another important example of the two-qubit operation is a CNOT-gate, which flips the state of the target qubit conditioned on the control qubit being in state $|1\rangle$, and a zero-controlled NOT-gate (Z-CNOT), which does the same provided that the control qubit is $|0\rangle$. Both these operations can be synthesized upon using several single- and double qubit gates; see Fig. 14 and Table I. We are particularly interested in these gates as they are required for realization of Deutsch's algorithm discussed in the following section. 
TABLE I. Definitions of the Oracles for the quantum Deutsch's algorithm. Here we use the following notation for the bit-flip operation $\mathcal{X}=\mathcal{R}_{x}(\pi)$.

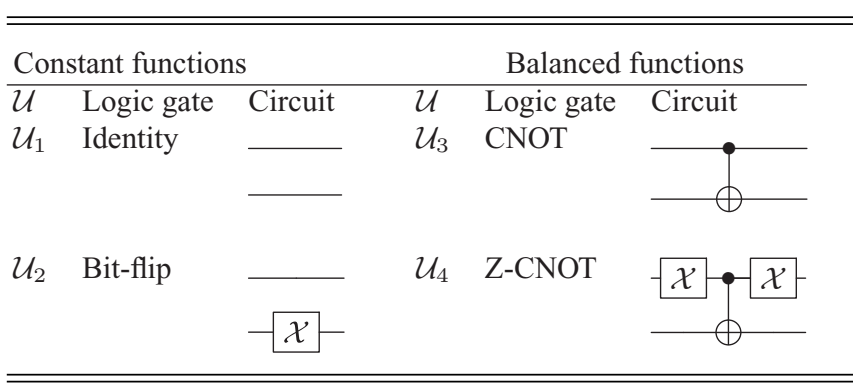

\section{APPENDIX I: IMPLEMENTATION OF DEUTSCH'S ALGORITHM}

Deutsch's algorithm is realized with the set of four twoqubit logic functions $\mathcal{U}_{i}$, whose identities are summarized in Table I. The gates corresponding to the constant functions are displayed in the left column. These include the identity gate $\mathcal{U}_{1}=\mathbb{1} \otimes \mathbb{1}$, which performs no operations between the preprocessing and the postprocessing Hadamard transforms of Deutsch's algorithm; see Fig. 8(a)] and the second qubit flip gate $\mathcal{U}_{2}=\mathbb{1} \otimes \mathcal{R}_{x}(\pi)$. The balanced functions $\mathcal{U}_{3}$ and $\mathcal{U}_{4}$ are encoded by the CNOT- and the zero-controlled NOT gates, respectively. The circuit shown in Fig. 8(a) operates with the input state $|\Psi\rangle=(0,1,0,0)^{T}$, i.e., the first qubit in the state $|0\rangle$ and the second qubit in the state $|1\rangle$. The algorithm works correctly if the output state of the first qubit is $|0\rangle$ for the gates $\mathcal{U}_{1}$ and $\mathcal{U}_{2}$, and $|1\rangle$ for the balanced gates $\mathcal{U}_{3}$ and $\mathcal{U}_{4}$.

For simulation of the Deutsch's algorithm implementation, we solve the following equation of motion for the four-component complex two-qubit state column-vector $|\Psi\rangle$, whose elements are the amplitudes of the states $|00\rangle,|01\rangle$, $|10\rangle$, and $|11\rangle$ :

$$
i \partial_{t}|\Psi\rangle=\hat{H}(t)|\Psi\rangle,
$$

where $\hat{H}(t)$ is given by Eqs. (E5) and (F4). The sequence of logic gates is encoded in the time dependence of the control parameters $\delta \omega_{1}(t)$ and $\delta \omega_{2}(t)$, which are regulated by means of the external voltage; see Fig. 15 . We assume

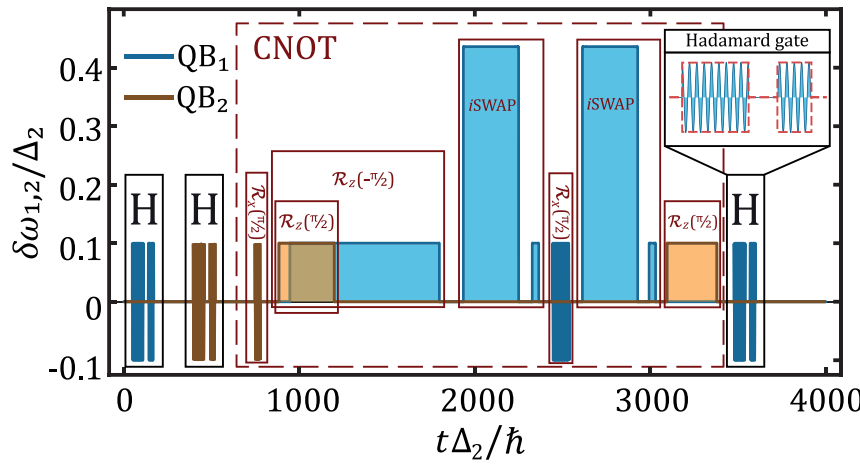

FIG. 15. The control pulse sequence which encodes a single run of the quantum Deutsch's algortihm with the oracle $\mathcal{U}_{3}$. The blue lines show the pulses applied to the first qubit $\left(\mathrm{QB}_{1}\right)$ while the orange lines correspond to the pulses governing the second qubit state $\left(\mathrm{QB}_{2}\right)$. The Hadamard gates $\mathbf{H}$ as well as the $\mathcal{R}_{x}$ operations are implemented by the AC pulses, whose carrier frequency is too high to be resolved at the given temporal scale. For clarification, the signal encoding the last Hadamard operation is zoomed in the inset; see also Fig. 13. The gap of the second qubit $\Delta_{2}$ is used as a normalization parameter for both axis grids.

that the amplitudes of the ac and dc electric pulses used for the implementation of local Pauli rotations are equal. These values correspond to the bias $\delta \omega_{0}$, which is considered as the governing parameter for testing the validity of the RWA in Fig. 8(b). The $i$ SWAP operations are realized by the square pulses which bring the qubits to the avoided crossing; see Fig. 7. The amplitude of these pulses is determined by the detuning, $\delta \omega_{0}=\sqrt{\Delta_{2}^{2}-\Delta_{1}^{2}}$. In our simulations we considered the qubits detuned such as $\Delta_{1} / \Delta_{2}=0.9$.

The strongest reduction of the overall algorithm fidelity is due to the limited validity of the rotating wave approximation assumed for the local Pauli gates. Since the gate operation duration is proportional to the electric field amplitude, the use of the weak electric field is undesirable. It makes longer the total run time of the algorithm, which is fundamentally limited from above by the finite coherence time.
[1] M. A. Nielsen and I. L. Chuang, Quantum Computation and Quantum Information (Cambridge University Press, New York, 2010).

[2] J. E. Mooij, T. P. Orlando, L. Levitov, L. Tian, C. H. Van der Wal, and S. Lloyd, Josephson persistent-current qubit, Science 285, 1036 (1999).

[3] T. Hime, P. A. Reichardt, B. L. T. Plourde, T. L. Robertson, C.-E. Wu, A. V. Ustinov, and J. Clarke, Solid-state qubits with current-controlled coupling, Science 314, 1427 (2006).

[4] J. Clarke and F. K. Wilhelm, Superconducting quantum bits, Nature (London) 453, 1031 (2008).

[5] K. G. Fedorov, A. V. Shcherbakova, M. J. Wolf, D. Beckmann, and A. V. Ustinov, Fluxon Readout of a Superconducting Qubit, Phys. Rev. Lett. 112, 160502 (2014).
[6] C. Neill, P. Roushan, K. Kechedzhi, S. Boixo, S. V. Isakov, V. Smelyanskiy, A. Megrant, B. Chiaro, A. Dunsworth, K. Arya et al., A blueprint for demonstrating quantum supremacy with superconducting qubits, Science 360, 195 (2018).

[7] F. Arute, K. Arya, R. Babbush, D. Bacon, J. C. Bardin, R. Barends, R. Biswas, S. Boixo, F. G. Brandao, D. A. Buell et al., Quantum supremacy using a programmable superconducting processor, Nature (London) 574, 505 (2019).

[8] D. Jaksch, C. Bruder, J. I. Cirac, C. W. Gardiner, and P. Zoller, Cold Bosonic Atoms in Optical Lattices, Phys. Rev. Lett. 81, 3108 (1998).

[9] H. Bernien, S. Schwartz, A. Keesling, H. Levine, A. Omran, H. Pichler, S. Choi, A. S. Zibrov, M. Endres, M. Greiner et al., Probing many-body dynamics on a 51-atom quantum simulator, Nature (London) 551, 579 (2017). 
[10] A. Keesling, A. Omran, H. Levine, H. Bernien, H. Pichler, S. Choi, R. Samajdar, S. Schwartz, P. Silvi, S. Sachdev et al., Quantum Kibble-Zurek mechanism and critical dynamics on a programmable Rydberg simulator, Nature (London) 568, 207 (2019).

[11] B. Lekitsch, S. Weidt, A. G. Fowler, K. Mølmer, S. J. Devitt, C. Wunderlich, and W. K. Hensinger, Blueprint for a microwave trapped ion quantum computer, Sci. Adv. 3, e1601540 (2017).

[12] J. Zhang, G. Pagano, P. W. Hess, A. Kyprianidis, P. Becker, H. Kaplan, A. V. Gorshkov, Z. Gong, and C. Monroe, Observation of a many-body dynamical phase transition with a 53-qubit quantum simulator, Nature (London) 551, 601 (2017).

[13] C. Kokail, C. Maier, R. van Bijnen, T. Brydges, M. K. Joshi, P. Jurcevic, C. A. Muschik, P. Silvi, R. Blatt, C. F. Roos et al., Self-verifying variational quantum simulation of lattice models, Nature (London) 569, 355 (2019).

[14] D. J. Brod, E. F. Galvão, A. Crespi, R. Osellame, N. Spagnolo, and F. Sciarrino, Photonic implementation of boson sampling: A review, Adv. Photon. 1, 034001 (2019).

[15] X. Zhang, H.-O. Li, G. Cao, M. Xiao, G.-C. Guo, and G.-P. Guo, Semiconductor quantum computation, Natl. Sci. Rev. 6, 32 (2019).

[16] D. M. Zajac, A. J. Sigillito, M. Russ, F. Borjans, J. M. Taylor, G. Burkard, and J. R. Petta, Resonantly driven CNOT gate for electron spins, Science 359, 439 (2018).

[17] J. Yoneda, K. Takeda, T. Otsuka, T. Nakajima, M. R. Delbecq, G. Allison, T. Honda, T. Kodera, S. Oda, Y. Hoshi, N. Usami, K. M. Itoh, and S. Tarucha, A quantum-dot spin qubit with coherence limited by charge noise and fidelity higher than $99.9 \%$, Nat. Nanotechnol. 13, 102 (2018).

[18] H. Wang, J. Qin, X. Ding, M.-C. Chen, S. Chen, X. You, Y.-M. He, X. Jiang, L. You, Z. Wang et al., Boson Sampling with 20 Input Photons and a 60-Mode Interferometer in a $10^{14}$-Dimensional Hilbert Space, Phys. Rev. Lett. 123, 250503 (2019).

[19] N. G. Berloff, M. Silva, K. Kalinin, A. Askitopoulos, J. D. Töpfer, P. Cilibrizzi, W. Langbein, and P. G. Lagoudakis, Realizing the classical $X Y$ Hamiltonian in polariton simulators, Nat. Mater. 16, 1120 (2017).

[20] P. G. Lagoudakis and N. G. Berloff, A polariton graph simulator, New J. Phys. 19, 125008 (2017).

[21] A. Delteil, T. Fink, A. Schade, S. Höfling, C. Schneider, and A. İmamoğlu, Towards polariton blockade of confined excitonpolaritons, Nat. Mater. 18, 219 (2019).

[22] S. Ghosh and T. C. H. Liew, Quantum computing with excitonpolariton condensates, npj Quantum Inf. 6, 16 (2020).

[23] I. Carusotto and C. Ciuti, Quantum fluids of light, Rev. Mod. Phys. 85, 299 (2013).

[24] A. J. Leggett, Macroscopic quantum systems and the quantum theory of measurement, Prog. Theor. Phys. Suppl. 69, 80 (1980).

[25] C. H. van der Wal, A. C. J. ter Haar, F. K. Wilhelm, R. N. Schouten, C. J. P. M. Harmans, T. P. Orlando, S. Lloyd, and J. E. Mooij, Quantum superposition of macroscopic persistentcurrent states, Science 290, 773 (2000).

[26] J. M. Martinis, S. Nam, J. Aumentado, and C. Urbina, Rabi Oscillations in a Large Josephson-Junction Qubit, Phys. Rev. Lett. 89, 117901 (2002).

[27] I. A. Shelykh, G. Pavlovic, D. D. Solnyshkov, and G. Malpuech, Proposal for a Mesoscopic Optical
Berry-Phase Interferometer, Phys. Rev. Lett. 102, 046407 (2009).

[28] A. Dreismann, P. Cristofolini, R. Balili, G. Christmann, F. Pinsker, N. G. Berloff, Z. Hatzopoulos, P. G. Savvidis, and J. J. Baumberg, Coupled counterrotating polariton condensates in optically defined annular potentials, Proc. Natl. Acad. Sci. USA 111, 8770 (2014).

[29] A. Amo, S. Pigeon, D. Sanvitto, V. G. Sala, R. Hivet, I. Carusotto, F. Pisanello, G. Leménager, R. Houdré, E. Giacobino et al., Polariton superfluids reveal quantum hydrodynamic solitons, Science 332, 1167 (2011).

[30] A. Muñoz Mateo, A. Gallemí, M. Guilleumas, and R. Mayol, Persistent currents supported by solitary waves in toroidal BoseEinstein condensates, Phys. Rev. A 91, 063625 (2015).

[31] A. Askitopoulos, H. Sigurdsson, I. Gnusov, S. Alyatkin, L. Pickup, N. A. Gippius, and P. Lagoudakis, Coherence revivals of a spinor polariton condensate from self-induced Larmor precession, arXiv:2006.01741 [cond-mat.quant-gas].

[32] H.-T. Lim, E. Togan, M. Kroner, J. Miguel-Sanchez, and A. Imamoğlu, Electrically tunable artificial gauge potential for polaritons, Nat. Commun. 8, 14540 (2017).

[33] I. L. Chuang, L. M. K. Vandersypen, X. Zhou, D. W. Leung, and S. Lloyd, Experimental realization of a quantum algorithm, Nature (London) 393, 143 (1998).

[34] J. A. Jones and M. Mosca, Implementation of a quantum algorithm on a nuclear magnetic resonance quantum computer, J. Chem. Phys. 109, 1648 (1998).

[35] S. Gulde, M. Riebe, G. P. T. Lancaster, C. Becher, J. Eschner, H. Häffner, F. Schmidt-Kaler, I. L. Chuang, and R. Blatt, Implementation of the Deutsch-Jozsa algorithm on an ion-trap quantum computer, Nature (London) 421, 48 (2003).

[36] L. DiCarlo, J. M. Chow, J. M. Gambetta, L. S. Bishop, B. R. Johnson, D. I. Schuster, J. Majer, A. Blais, L. Frunzio, S. M. Girvin, and R. J. Schoelkopf, Demonstration of two-qubit algorithms with a superconducting quantum processor, Nature (London) 460, 240 (2009).

[37] M. Mohseni, J. S. Lundeen, K. J. Resch, and A. M. Steinberg, Experimental Application of Decoherence-Free Subspaces in an Optical Quantum-Computing Algorithm, Phys. Rev. Lett. 91, 187903 (2003).

[38] M. S. Tame, R. Prevedel, M. Paternostro, P. Böhi, M. S. Kim, and A. Zeilinger, Experimental Realization of Deutsch's Algorithm in a one-Way Quantum Computer, Phys. Rev. Lett. 98, 140501 (2007).

[39] S. Eckel, F. Jendrzejewski, A. Kumar, C. J. Lobb, and G. K. Campbell, Interferometric Measurement of the Current-Phase Relationship of a Superfluid Weak Link, Phys. Rev. X 4, 031052 (2014).

[40] C. Zhang and G. Jin, Rotation of exciton-polariton condensates with TE-TM splitting in a microcavity ring, New J. Phys. 19, 093002 (2017).

[41] L. Amico, D. Aghamalyan, F. Auksztol, H. Crepaz, R. Dumke, and L. C. Kwek, Superfluid qubit systems with ring shaped optical lattices, Sci. Rep. 4, 4298 (2014).

[42] Y. Xue, Y. Jiang, G. Wang, R. Wang, S. Feng, and M. Matuszewski, Creation of stable dark and anti-dark solitons in polariton dyad, Opt. Express 26, 6267 (2018).

[43] P. D. Drummond, A. Eleftheriou, K. Huang, and K. V. Kheruntsyan, Theory of a mode-locked atom laser with toroidal geometry, Phys. Rev. A 63, 053602 (2001). 
[44] F. Tassone and Y. Yamamoto, Exciton-exciton scattering dynamics in a semiconductor microcavity and stimulated scattering into polaritons, Phys. Rev. B 59, 10830 (1999).

[45] G. Roumpos, M. D. Fraser, A. Löffler, S. Höfling, A. Forchel, and Y. Yamamoto, Single vortex-antivortex pair in an excitonpolariton condensate, Nat. Phys. 7, 129 (2011).

[46] M. Wouters and V. Savona, Stochastic classical field model for polariton condensates, Phys. Rev. B 79, 165302 (2009).

[47] E. J. Mueller, Superfluidity and mean-field energy loops: Hysteretic behavior in Bose-Einstein condensates, Phys. Rev. A 66, 063603 (2002).

[48] A. V. Nalitov, H. Sigurdsson, S. Morina, Y. S. Krivosenko, I. V. Iorsh, Y. G. Rubo, A. V. Kavokin, and I. A. Shelykh, Optically trapped polariton condensates as semiclassical time crystals, Phys. Rev. A 99, 033830 (2019).

[49] X. Ma, I. Y. Chestnov, M. V. Charukhchyan, A. P. Alodjants, and O. A. Egorov, Oscillatory dynamics of nonequilibrium dissipative exciton-polariton condensates in weak-contrast lattices, Phys. Rev. B 91, 214301 (2015).

[50] See Supplemental Material at http://link.aps.org/supplemental/ 10.1103/PhysRevResearch.3.013099 for the movie of the polariton condensate dynamics.

[51] G. Liu, D. W. Snoke, A. Daley, L. N. Pfeiffer, and K. West, A new type of half-quantum circulation in a macroscopic polariton spinor ring condensate, Proc. Natl. Acad. Sci. USA 112, 2676 (2015).

[52] V. A. Lukoshkin, V. K. Kalevich, M. M. Afanasiev, K. V. Kavokin, Z. Hatzopoulos, P. G. Savvidis, E. S. Sedov, and A. V. Kavokin, Persistent circular currents of exciton-polaritons in cylindrical pillar microcavities, Phys. Rev. B 97, 195149 (2018).

[53] E. S. Sedov, V. A. Lukoshkin, V. K. Kalevich, P. G. Savvidis, and A. V. Kavokin, Circular polariton currents with integer and fractional orbital angular momenta, Phys. Rev. Research 3, 013072 (2021).

[54] Edited by G. Farin, J. Hoschek, and M. S. Kim, Handbook of Computer Aided Geometric Design (Elsevier, Amsterdam, 2002).

[55] S. Mukherjee, D. M. Myers, R. G. Lena, B. Ozden, J. Beaumariage, Z. Sun, M. Steger, L. N. Pfeiffer, K. West, A. J. Daley, and D. W. Snoke, Observation of nonequilibrium motion and equilibration in polariton rings, Phys. Rev. B 100, 245304 (2019).

[56] E. Sedov, V. Lukoshkin, V. Kalevich, Z. Hatzopoulos, P. Savvidis, and A. Kavokin, Persistent currents in half-moon polariton condensates, ACS Photonics 7, 1163 (2020).

[57] A. Bezryadina, E. Eugenieva, and Z. Chen, Self-trapping and flipping of double-charged vortices in optically induced photonic lattices, Opt. Lett. 31, 2456 (2006).

[58] A. Oliinyk, A. Yakimenko, and B. Malomed, Tunneling of persistent currents in coupled ring-shaped Bose-Einstein condensates, J. Phys. B 52, 225301 (2019).

[59] Z. Chen, Y. Li, and B. A. Malomed, Josephson oscillations of chirality and identity in two-dimensional solitons in spin-orbitcoupled condensates, Phys. Rev. Res. 2, 033214 (2020).

[60] A. J. Ramsay, A. V. Gopal, E. M. Gauger, A. Nazir, B. W. Lovett, A. M. Fox, and M. S. Skolnick, Damping of Exciton Rabi Rotations by Acoustic Phonons in Optically Excited InGaAs/GaAs Quantum Dots, Phys. Rev. Lett. 104, 017402 (2010).
[61] M. V. Gustafsson, T. Aref, A. F. Kockum, M. K. Ekström, G. Johansson, and P. Delsing, Propagating phonons coupled to an artificial atom, Science 346, 207 (2014).

[62] J. L. Skinner and D. Hsu, Pure dephasing of a two-level system, J. Phys. Chem. 90, 4931 (1986).

[63] D. M. Whittaker and P. R. Eastham, Coherence properties of the microcavity polariton condensate, Europhys. Lett. 87, 27002 (2009).

[64] A. Askitopoulos, L. Pickup, S. Alyatkin, A. Zasedatelev, K. G. Lagoudakis, W. Langbein, and P. G. Lagoudakis, Giant increase of temporal coherence in optically trapped polariton condensate, arXiv:1911.08981.

[65] P. Lagoudakis and A. Askitopoulos (private communication).

[66] L. Dominici, D. Colas, S. Donati, J. P. Restrepo Cuartas, M. De Giorgi, D. Ballarini, G. Guirales, J. C. López Carreño, A. Bramati, G. Gigli et al., Ultrafast Control and Rabi Oscillations of Polaritons, Phys. Rev. Lett. 113, 226401 (2014).

[67] I. Y. Chestnov, A. V. Kavokin, and A. V. Yulin, The optical control of phase locking of polariton condensates, New J. Phys. 21, 113009 (2019).

[68] D. G. Thomas and J. J. Hopfield, A magneto-Stark effect and exciton motion in CdS, Phys. Rev. 124, 657 (1961).

[69] D. C. McKay, C. J. Wood, S. Sheldon, J. M. Chow, and J. M. Gambetta, Efficient $Z$ gates for quantum computing, Phys. Rev. A 96, 022330 (2017).

[70] T. C. H. Liew, A. V. Kavokin, and I. A. Shelykh, Optical Circuits Based on Polariton Neurons in Semiconductor Microcavities, Phys. Rev. Lett. 101, 016402 (2008).

[71] D. P. DiVincenzo, D. Bacon, J. Kempe, G. Burkard, and K. B. Whaley, Universal quantum computation with the exchange interaction, Nature (London) 408, 339 (2000).

[72] N. Schuch and J. Siewert, Natural two-qubit gate for quantum computation using the XY interaction, Phys. Rev. A 67, 032301 (2003).

[73] A. C. Mathey and L. Mathey, Realizing and optimizing an atomtronic SQUID, New J. Phys. 18, 055016 (2016).

[74] D. Deutsch and R. Jozsa, Rapid solution of problems by quantum computation, Proc. R. Soc. London A 439, 553 (1992).

[75] K. G. Lagoudakis, B. Pietka, M. Wouters, R. André, and B. Deveaud-Plédran, Coherent Oscillations in an ExcitonPolariton Josephson Junction, Phys. Rev. Lett. 105, 120403 (2010).

[76] K. Rayanov, B. L. Altshuler, Y. G. Rubo, and S. Flach, Frequency Combs with Weakly Lasing ExcitonPolariton Condensates, Phys. Rev. Lett. 114, 193901 (2015).

[77] S. Kim, Y. G. Rubo, T. C. H. Liew, S. Brodbeck, C. Schneider, S. Höfling, and H. Deng, Emergence of microfrequency comb via limit cycles in dissipatively coupled condensates, Phys. Rev. B 101, 085302 (2020).

[78] L. P. Gorkov and I. E. Dzyaloshinskii, Contribution to the theory of the Mott exciton in a strong magnetic field, Sov. Phys. JETP 26, 449 (1968).

[79] S. I. Shevchenko, Vortices and vortical structures in systems with pairing of spatially separated electrons and holes, Phys. Rev. B 56, 10355 (1997).

[80] P. Krantz, M. Kjaergaard, F. Yan, T. P. Orlando, S. Gustavsson, and W. D. Oliver, A quantum engineer's guide to superconducting qubits, Appl. Phys. Rev. 6, 021318 (2019). 